\title{
Variable slice thickness (VAST) EPI for the reduction of susceptibility artifacts in whole-brain GE-EPI at 7 Tesla
}

\author{
Citation for published version (APA):
}

Brunheim, S., Johst, S., Pfaffenrot, V., Maderwald, S., Quick, H. H., \& Poser, B. A. (2017). Variable slice thickness (VAST) EPI for the reduction of susceptibility artifacts in whole-brain GE-EPI at 7 Tesla. Magnetic Resonance Materials in Physics Biology and Medicine, 30(6), 591-607. https://doi.org/10.1007/s10334-017-0641-0

Document status and date:

Published: 01/12/2017

DOI:

10.1007/s10334-017-0641-0

\section{Document Version:}

Publisher's PDF, also known as Version of record

\section{Document license:}

Taverne

\section{Please check the document version of this publication:}

- A submitted manuscript is the version of the article upon submission and before peer-review. There can be important differences between the submitted version and the official published version of record.

People interested in the research are advised to contact the author for the final version of the publication, or visit the DOI to the publisher's website.

- The final author version and the galley proof are versions of the publication after peer review.

- The final published version features the final layout of the paper including the volume, issue and page numbers.

Link to publication

\footnotetext{
General rights rights.

- You may freely distribute the URL identifying the publication in the public portal. please follow below link for the End User Agreement:

www.umlib.nl/taverne-license

Take down policy

If you believe that this document breaches copyright please contact us at:

repository@maastrichtuniversity.nl

providing details and we will investigate your claim.
}

Copyright and moral rights for the publications made accessible in the public portal are retained by the authors and/or other copyright owners and it is a condition of accessing publications that users recognise and abide by the legal requirements associated with these

- Users may download and print one copy of any publication from the public portal for the purpose of private study or research.

- You may not further distribute the material or use it for any profit-making activity or commercial gain

If the publication is distributed under the terms of Article $25 \mathrm{fa}$ of the Dutch Copyright Act, indicated by the "Taverne" license above, 


\title{
Variable slice thickness (VAST) EPI for the reduction of susceptibility artifacts in whole-brain GE-EPI at 7 Tesla
}

\author{
Sascha Brunheim ${ }^{1,2}$ (i) $\cdot$ Sören Johst ${ }^{1} \cdot$ Viktor Pfaffenrot $^{1,2} \cdot$ Stefan Maderwald $^{1}$ • \\ Harald H. Quick ${ }^{1,2} \cdot$ Benedikt A. Poser $^{3}$
}

Received: 23 March 2017 / Revised: 23 June 2017 / Accepted: 26 June 2017 / Published online: 10 July 2017

(C) ESMRMB 2017

\begin{abstract}
Objective A new technique for 2D gradient-recalled echo echo-planar imaging (GE-EPI) termed 'variable slice thickness' (VAST) is proposed, which reduces signal losses caused by through-slice susceptibility artifacts, while keeping the volume repetition time (TR) manageable. The slice thickness is varied across the brain, with thinner slices being used in the inferior brain regions where signal voids are most severe.

Materials and methods Various axial slice thickness schemes with identical whole-brain coverage were compared to regular EPI, which may either suffer from unfeasibly long TR if appropriately thin slices are used throughout, or signal loss if no counter-measures are taken. Evaluation is based on time-course signal-to-noise (tSNR) maps from resting state data and a statistical group-level region of interest (ROI) analysis on breath-hold fMRI measurements. Results The inferior brain region signal voids with static B0 inhomogeneities could be markedly reduced with VAST GE-EPI in contrast to regular GE-EPI. ROI-averaged
\end{abstract}

Electronic supplementary material The online version of this article (doi:10.1007/s10334-017-0641-0) contains supplementary material, which is available to authorized users.

Sascha Brunheim

sascha.brunheim@uni-due.de

1 Erwin L. Hahn Institute for Magnetic Resonance Imaging, University of Duisburg-Essen, Kokereiallee 7, Building C84, 45141 Essen, Germany

2 High Field and Hybrid MR Imaging, University Hospital Essen, Hufelandstrasse 55, 45147 Essen, Germany

3 Department of Cognitive Neuroscience, Faculty of Psychology and Neuroscience, Maastricht Brain Imaging Centre, Maastricht University, PO Box 616, 6200 MD Maastricht, The Netherlands event-related signal changes showed $48 \%$ increase in VAST compared to GE-EPI with regular "thick" slices. tSNR measurements proved the comparable signal robustness of VAST in comparison to regular GE-EPI with thin slices.

Conclusion A novel acquisition strategy for functional 2D GE-EPI at ultrahigh magnetic field is presented to reduce susceptibility-induced signal voids and keep TR sufficiently short for whole-brain coverage.

Keywords fMRI $\cdot$ Echo-planar imaging $\cdot$ Susceptibility artifact $\cdot$ Repetition time $\cdot$ Slice thickness $\cdot 7$ Tesla ultrahigh field MRI

\section{Introduction}

Functional magnetic resonance imaging (fMRI) for the purpose of studying neuronal activity in human brain relies heavily on the gradient-recalled echo echo-planar imaging (GE-EPI) technique. This method maps signal variations induced by changes in the non-refocused spin-spin relaxation time $\left(T_{2}^{*}\right)$ that accompany neuronal activation. The different magnetic properties of oxyhemoglobin and deoxyhemoglobin [1] cause main magnetic field (B0) distortions within and around the venous blood vessels and capillaries [2]. These lead to locally measurable signal fluctuations known as the blood oxygenation level-dependent (BOLD) effect [3]. Furthermore, the signal intensity scales quadratically with B0 such that ultrahigh field (UHF) fMRI benefits from this effect [4]. While $T_{2}^{*}$-weighted fMRI provides the most sensitive tool for mapping the desired BOLDrelated B0 effects that occur at the microscopic level, the technique is inevitably also sensitive to macroscopic B0 inhomogeneities [5]. The problem is most pronounced near the skull's air cavities (mouth, sinuses, ear canals) and 
compromises BOLD fMRI in brain regions including the orbitofrontal cortex (OFC), ventromedial prefrontal cortex (vmPFC), and inferior temporal gyrus (ITG) [6].

In EPI, where the phase-encoding bandwidth is low, any in-plane component of the undesired field gradient will cause the well-known geometric distortions along the phase-encode direction [7,8], irrespective of $T_{2}^{*}$ and $T_{2}$ contrast. For a commonly used axial slice orientation, the through-plane component $G_{\mathrm{S}, z}$ of the susceptibility gradient $G_{\mathrm{S}}$ leads to severe signal dropout in gradient-echo acquisition [9] because of spin dephasing across the slice profile. As a consequence, this artifact is absent in spin-echo acquisition where any such spin dephasing is refocused at the echo time (TE).

Mathematically, the phenomenon of the through-plane signal voids in GE sequences can be written with signal $\mathfrak{S}$ [10], originating from a voxel at position $r$ and an initial longitudinal magnetization $M_{0}$ with the gyromagnetic ratio $\gamma$ in the absence of susceptibility and any shimming gradients as

$$
\begin{aligned}
& \mathfrak{S}(\vec{r}) \propto M_{0}(\vec{r}) \int_{-\Delta z_{/ 2}}^{\Delta z / 2} \frac{z}{\Delta z} \exp \left[-\mathrm{i} \gamma G_{\mathrm{S}, z}(\vec{r}) z \mathrm{TE}\right] \mathrm{d} z \\
& =M_{0}(\vec{r}) \operatorname{sinc}\left[\frac{\gamma}{2} G_{\mathrm{S}, z}(\vec{r}) \Delta z \mathrm{TE}\right]
\end{aligned}
$$

The term 'sinc' describes the excitation pulse form in time which translates into a rectangular slice excitation profile in the frequency domain after Fourier transformation. The exponential $T_{2}^{*}$-weighted signal decay is hence modulated by a sinc term that depends on the slice thickness $\Delta z$, $\mathrm{TE}$, and the magnitude of the through-plane component of the susceptibility-induced B0 gradient $G_{\mathrm{S}, z}$. This formalism will be revisited below.

Correction for the through-plane signal void artifact is non-trivial due to the localized nature of the susceptibility changes, and anatomical variations from subject to subject [11]. The artifact also presents a particular challenge for UHF fMRI (7 $\mathrm{T}$ and higher) as the susceptibility-induced gradient scales with B0.

Over the past two decades, a large number of different methods have been proposed for mitigating the susceptibility-induced signal voids in inferior brain regions. These include choice of optimal slice orientation [11, 12], or thinner slices for a defined brain region (e.g. amygdala) [13], z-shimming method [9, 14-16], slice-dependent TE adjustment [17], passive radiofrequency (RF) shimming by dielectric inlays or bags [18-20], use of tailored 3D/4D RF pulses [21-23], and parallel RF excitation concepts
[24-26]. However, each of these methods also have disadvantages for functional imaging with whole-brain coverage in an appropriate sampling time. For instance, a prior adjustment of the 3D or 4D RF pulse form with a certain bandwidth $\Delta f$ is necessary for every individual subject $[22,23]$. The optimization of RF pulse parameters will compromise sensitivity elsewhere in the brain and in vivo specific absorption rate (SAR) constraints have to be considered. The use of $\mathrm{z}$-shimming in order to re-phase the through-plane signal loss will only precisely match the $G_{\mathrm{S}}$ in a specific location. Additionally, one has to record each slice multiple times with different amounts of gradient compensation which can become a time-consuming process that reduces the effective temporal resolution of the fMRI experiment. The scope for reduction of TE $\leq T_{2}^{*}$ to compensate for the sinc term in Eq. 2 in order to achieve optimal BOLD sensitivity in inferior brain regions [17] is reduced at higher field strengths. Here, typically shorter TE is required due to the shorter $T_{2}^{*}$, but the duration of the EPI readout itself becomes the limiting factor, especially if a large matrix is acquired at UHF. Furthermore, the values for TE and $T_{2}^{*}$ are reduced even more if the examined slice is located near the cranial base [27, 28]. Using thinner slices for investigation of a localized region above the base of the skull, like the amygdala [13], does not provide information about neuronal activity within the rest of the brain. In comparison to the z-shimming method, a simple reduction of the slice thickness is much easier to implement for the susceptibility-induced artifact compensation in 2D GE-EPI [29].

In summary, it could be worthwhile for a 2D GE-EPI acquisition to vary the slice thickness $\Delta z$ across the brain in order to reduce signal voids where they are present (inferior brain regions) while using thicker slices in the unaffected superior brain regions. In this way, one will not unnecessarily compromise the overall SNR where no susceptibility artifact compensation is required. At least as importantly, a variable slice thickness approach will help avoid unfeasibly long volume repetition times (TRs) compared to a typical approach that uses thinner slices for the entire brain. The present work investigates three different variable slice thickness (VAST) GE-EPI schemes designed to compensate for the inferior brain region through-slice signal dropout.

\section{Materials and methods}

\section{VAST GE-EPI concept}

It can be seen from rewriting Eq. 2 that for a rectangular slice excitation in the $z$-direction, increasing $G_{z}$ to compensate for $G_{\mathrm{S}, z}$ and/or reducing TE or the RF pulse bandwidth 
$\Delta f=\gamma G_{z} \Delta z / 2 \pi$ has a direct effect on the local signal intensity

$$
\mathfrak{S}(\vec{r}) \propto M_{0}(\vec{r}) \operatorname{sinc}\left[\frac{\gamma}{2} G_{\mathrm{S}, z}(\vec{r}) \Delta z \mathrm{TE}\right] \quad \text {, with } \Delta z=\frac{2 \pi \Delta f}{\gamma G_{z}}
$$

$=M_{0}(\vec{r}) \operatorname{sinc}\left[\pi G_{\mathrm{S}, z}(\vec{r}) \frac{\Delta f}{G_{z}} \mathrm{TE}\right]$

To achieve a given reduction in slice thickness $\Delta z$, Eq. 4 shows two possibilities decrease $\Delta f$ for a given slice selection gradient strength $G_{z}$, or alternatively increase $G_{z}$, while keeping $\Delta f$ the same. For practical implementation in the sequence, the latter is preferred. Hence, for VAST, the different slice thicknesses, positions, and the inverse proportional scaling factors can be prepared straightforward with respect to the global sequence parameters chosen by the operator and are applied during runtime, rather than defining RF pulse objects with a different bandwidth each.

\section{Noise distribution for different slice thicknesses}

In a time-series measurement, the locally observed noise $\sigma$ $(\vec{r})$ can be described as the combination of Gaussian distributed thermal $\sigma_{0}(\vec{r})$ and physiological noise $\sigma_{\mathrm{p}}(\vec{r})$, while the later scales with the image intensity $\mathfrak{S}$ [30]. This leads to the time-course signal-to-noise ratio (tSNR) for the mean signal $\overline{\mathfrak{S}}$ over time [31]:

$\operatorname{tSNR}(\vec{r})=\frac{\overline{\mathfrak{S}}(\vec{r})}{\sqrt{\sigma_{0}^{2}(\vec{r})+\sigma_{\mathrm{p}}^{2}(\vec{r})}}=\frac{\overline{\mathfrak{S}}(\vec{r})}{\sigma(\vec{r})}$

For high-resolution 2D GE-EPI at 7 Tesla, the physiological-to-thermal noise ratio $\sigma_{\text {ratio }}=\sigma_{\mathrm{p}} / \sigma_{0}$ is found to be approximately equal to one for an iso-voxel resolution of $V=(1.5 \mathrm{~mm})^{3}$, whilst it doubles for $(3 \mathrm{~mm})^{3}$ so that the measurements in this study are within the physiological noise-dominated regime [31]. Furthermore, it has been shown that acquiring high spatial resolution data followed by a smoothing operation improves the SNR without adding physiological noise $[32,33]$.

\section{MRI system and scanning procedure}

Data was acquired on a Siemens 7T UHF MRI system (MAGNETOM 7T, Siemens Healthcare GmbH, Erlangen, Germany) equipped with a large field of view (FOV) gradient coil (type AS095D, maximum gradient amplitude $28 \mathrm{mT} / \mathrm{m}$, maximum slew rate $170 \mathrm{mT} / \mathrm{m} / \mathrm{ms}$ ). The vendorprovided 1-channel transmit/32-channel receive RF head coil (Nova Medical Inc., Wilmington, DE, USA) was used in all experiments. Human data were acquired after obtaining informed consent and in accordance with local IRB approval. The general in vivo scanning procedure included a previous first- and second-order B0 shim for the wholebrain volume [34] and nominal transmitter voltage adjustment for a central brain region above the basal ganglia, based on pre-saturation turbo fast low angle shot (pre-sat TFL) flip angle mapping [35].

At the start of each session, an anatomical scan was acquired using an in-house variant of the MPRAGE sequence [36] with an adiabatic WURST RF pulse [37, 38]. The 3D MPRAGE sequence parameters were isotropic $0.7 \mathrm{~mm}$ voxels (matrix size $=384 \times 336 \times 256$ ), $\mathrm{TI}=1.1 \mathrm{~s}$, $\mathrm{TR}=2.5 \mathrm{~s}, \mathrm{TE}=1.54 \mathrm{~ms}$, GRAPPA acceleration factor $R=2$ with 48 ACS lines, and total acquisition time (TA) of approximately $6 \mathrm{~min}$. Subsequently, several different GEEPI sequence variants were conducted with a TA of under 5 min each (Table 1). These sequence variants were evaluated in combination with a breath-hold fMRI paradigm $(N=12)$ and additional resting state fMRI measurements for one subject tSNR map calculation were performed. Moreover, for the 2-block VAST-GE-EPI variant and the $1.5 \mathrm{~mm}$ control GE-EPI, normalized tSNR maps were calculated within the framework of a finger tapping fMRI experiment $(N=14)$. Further on, a description of the different sequence variants is given and its difference to the unvarying slice thickness of two control measurements.

\section{VAST GE-EPI variants}

A standard GE-EPI sequence (Fig. 1a) was modified to support three different VAST EPI variants (Fig. 1b-d) in order to accomplish a $\Delta z$ variation within the whole neocortex volume. Therefore, the slice selection gradient amplitude $G_{z}$ and its corresponding refocusing gradient were dynamically adjusted for each slice during sequence runtime [39]. For this implementation, the different EPI slices were acquired in linear ascending order from the skull base to vertex, while each slice position was adjusted accordingly. The 7T MRI system image reconstruction software (VB17) was modified to adjust the slice signal intensities in inverse proportion to the slice thickness, to keep signal intensity in the VAST scans homogenous over the whole brain, despite the differing acquisition voxel sizes. The in vivo EPI sequences included fat saturation by a non-selective Gaussian pulse prior to each slice excitation. This decreased the fat-water shift artifacts but also increased the overall TR by $21 \%$.

The EPI imaging parameters are summarized in Table 1, and noise ratios were given based on [31]. Specifically, the three different VAST GE-EPI methods consisted of: A 2-block design (Fig. 1b, green column in 
Table $12 \mathrm{D}$ sequence parameters used for both the normal GE-EPI versions with an uniform slice thickness $\Delta \mathrm{z}$ of $1.5 \mathrm{~mm}$ (grey column) and $3 \mathrm{~mm}$ (yellow column) for the breath-hold fMRI experiment (1st study), plus the different VAST-GE-EPI techniques with two blocks (green column), three blocks (blue column) and two blocks with a linear upward continuously increased $\Delta \mathrm{z}$ within the first block (red column). The values in brackets, marked with a dagger, represent
tSNR measurement parameters for $3 \mathrm{~mm}$ control and the three VAST variants of the 1st study which are different to the fMRI experiment. Whereas the values in brackets with a double dagger stand for the tSNR measurement parameters for $1.5 \mathrm{~mm}$ control and 2-block VAST of the 2nd study. The variant names and color coding shown here is employed further on for each figure and table to clearly assign the method used

\begin{tabular}{|c|c|c|c|c|c|}
\hline \multirow[b]{2}{*}{ (VAST-)GE-EPI variant name } & \multicolumn{2}{|c|}{ Control GE-EPIs } & \multicolumn{3}{|c|}{ VAST-GE-EPIs } \\
\hline & $1.5 \mathrm{~mm}$ & $3 \mathrm{~mm}$ & 2-block & 3-block & Linear \\
\hline Total slices & $67\left(70^{\dagger}, 91^{\ddagger}\right)$ & $34\left(35^{\dagger}\right)$ & $45\left(35^{\dagger}, 64^{\ddagger}\right)$ & $45\left(35^{\dagger}\right)$ & $39\left(35^{\dagger}\right)$ \\
\hline No. of volumes & $79\left(75^{\dagger}, 100^{\ddagger}\right)$ & $160\left(150^{\dagger}\right)$ & $120\left(150^{\dagger}, 100^{\ddagger}\right)$ & $120\left(150^{\dagger}\right)$ & $139\left(150^{\dagger}\right)$ \\
\hline Slice thickness [mm] & 1.5 & 3 & $1.5 / 3$ & $1.5 / 2.25 / 3$ & $1.5-3$ \\
\hline Slice distance factor [\%] & 0 & 0 & 0 & 0 & 0 \\
\hline TA $[\mathrm{s}]$ & $281\left(422^{\ddagger}\right)$ & $280\left(271^{\dagger}\right)$ & $280\left(271^{\dagger}, 296^{\ddagger}\right)$ & $280\left(271^{\dagger}\right)$ & $281\left(271^{\dagger}\right)$ \\
\hline $\operatorname{TR}_{\text {volume }}[\mathrm{s}]$ & $3.32\left(3.5^{\dagger}, 4^{\ddagger}\right)$ & $1.69\left(1.75^{\dagger}\right)$ & $2.23\left(1.75^{\dagger}, 2.8^{\ddagger}\right)$ & $2.23\left(1.75^{\dagger}\right)$ & $1.94\left(1.75^{\dagger}\right)$ \\
\hline TE [ms] & $20\left(22^{\ddagger}\right)$ & 20 & $20\left(22^{\ddagger}\right)$ & 20 & 20 \\
\hline Echo spacing [ms] & $0.76\left(0.87^{\ddagger}\right)$ & 0.76 & $0.76\left(0.87^{\ddagger}\right)$ & 0.76 & 0.76 \\
\hline Flip angle [deg] & $81\left(82^{\dagger}, 84^{\ddagger}\right)$ & $67\left(68^{\dagger}\right)$ & $73\left(68^{\dagger}, 78^{\ddagger}\right)$ & $73\left(68^{\dagger}\right)$ & $70\left(68^{\dagger}\right)$ \\
\hline Pulse length $[\mu \mathrm{s}]$ & $3072\left(3840^{\ddagger}\right)$ & 3072 & $3072\left(3840^{\ddagger}\right)$ & 3072 & 3072 \\
\hline Receiver bandwidth $[\mathrm{Hz} / \mathrm{Px}]$ & $1563\left(1302^{\ddagger}\right)$ & 1563 & $1563\left(1302^{\ddagger}\right)$ & 1563 & 1563 \\
\hline FOV $\left[\mathrm{mm}^{2}\right]$ & $192 \times 192$ & $192 \times 192$ & $192 \times 192$ & $192 \times 192$ & $192 \times 192$ \\
\hline Matrix size & $128 \times 128$ & $128 \times 128$ & $128 \times 128$ & $128 \times 128$ & $128 \times 128$ \\
\hline Partial Fourier factor & $6 / 8\left(7 / 8^{\ddagger}\right)$ & $6 / 8$ & $6 / 8\left(7 / 8^{\ddagger}\right)$ & $6 / 8$ & $6 / 8$ \\
\hline GRAPPA acceleration factor & 3 & 3 & 3 & 3 & 3 \\
\hline ACS lines & 48 & 48 & 48 & 48 & 48 \\
\hline
\end{tabular}

Table 1) in which the caudal half of the volume had a $\Delta z=1.5 \mathrm{~mm}\left(V=3.38 \mathrm{~mm}^{3}\right.$ and $\left.\sigma_{\text {ratio }}=1\right)$ and the cranial part had $\Delta z=3 \mathrm{~mm}\left(V=6.75 \mathrm{~mm}^{3}\right.$ and $\left.\sigma_{\text {ratio }}=1.12\right)$. For the 3-block variant (Fig. 1c, blue column in Table 1), the total volume was divided into three sections. Here, the slice thicknesses were $\Delta z=1.5 \mathrm{~mm}$ in the caudal quarter, $\Delta z=2.25 \mathrm{~mm}\left(V=5.06 \mathrm{~mm}^{3}\right.$, and $\left.\sigma_{\text {ratio }}=1.06\right)$ for the second quarter, and $\Delta z=3 \mathrm{~mm}$ for the remaining half of the volume. The linear VAST GE-EPI method is depicted in Fig. 1d with its sequence parameters also listed in Table 1 (red column). Within the caudal half of the total axial slice volume, the slice thickness was linearly increased from slice 1 with $\Delta z=1.5 \mathrm{~mm}$ to slice 20 with $\Delta z=2.96 \mathrm{~mm}$. Again, the second cranial part had an overall slice thickness of $\Delta z=3 \mathrm{~mm}$. Each of these protocols resulted in a total coverage of $\sim 100 \mathrm{~mm}(\sim 120 \mathrm{~mm}$ for the 2nd study, including the cerebellum) in the head-foot direction which we considered 'whole-neocortex coverage' ('whole-brain coverage' for the 2nd study).

For comparison to these VAST sequence variants, two different standard GE-EPI measurements were conducted which served as references (Fig. 1a), in the following, referred to as 'control'. A slice thickness of $3 \mathrm{~mm}$ throughout required 34 slices, resulting in the shortest $\mathrm{TR}=1.69 \mathrm{~s}$ for the same volume coverage (Table 1, yellow column). Doubling the number of slices for the $1.5-\mathrm{mm}$ experiment resulted in the longest TR $=3.32 \mathrm{~s}$ (Table 1 , grey column).
By contrast, the VAST GE-EPI protocols had a volume TR of only around $2 \mathrm{~s}$.

Figure 2A shows parts of the VAST GE-EPI sequence timing, with focus on the $G_{z}$ timing and amplitudes in relation to the RF pulse. From left to right: 10 separate slice excitations in ascending order are depicted from top to bottom for the 2-block (green), 3-block (blue), and linear VAST variant (red). For validation of the rectangular slice excitation profiles, measurements of a standard spherical head phantom were conducted which illustrate the different $\Delta z$ (Fig. 2b).

For verification of the VAST GE-EPI method's $\Delta z$ variation performance, previous phantom measurements with a spherical polydimethylsiloxane (PDMS) oil phantom (diameter $=165 \mathrm{~mm}, \mathrm{~T} 1=1150 \mathrm{~ms}, \mathrm{~T} 2=750 \mathrm{~ms})$ were conducted. The signal intensity results, shown in Supporting Figs. 1 and 2, proved the correct re-positioning and rescaling of the thinner slices before and after interpolation onto a general voxel resolution, which will be described within the following paragraph.

\section{General post-processing}

Twelve healthy volunteers (4 female) with a mean age of 28.8 years $(\mathrm{SD}=3.5)$ with normal or corrected to normal vision participated in the first study. In order to create a comparable basis, all in vivo datasets were re-sampled 
a

\section{$3 \mathrm{~mm}(1.5 \mathrm{~mm})$ control}

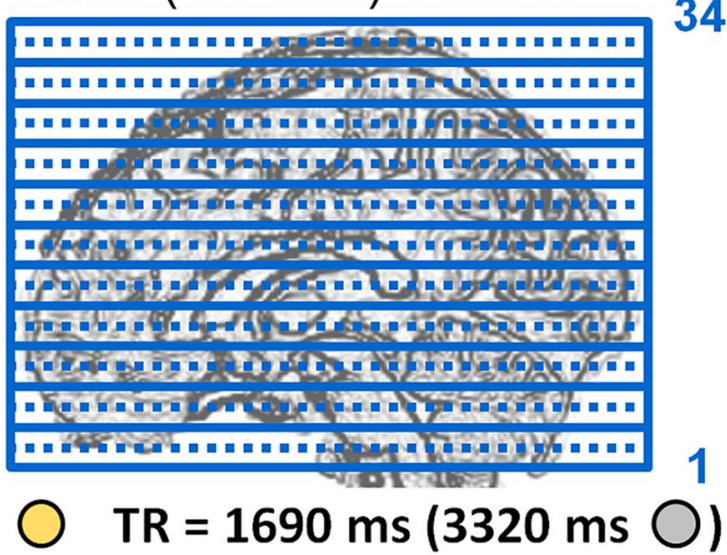

C

\section{3-block variant}

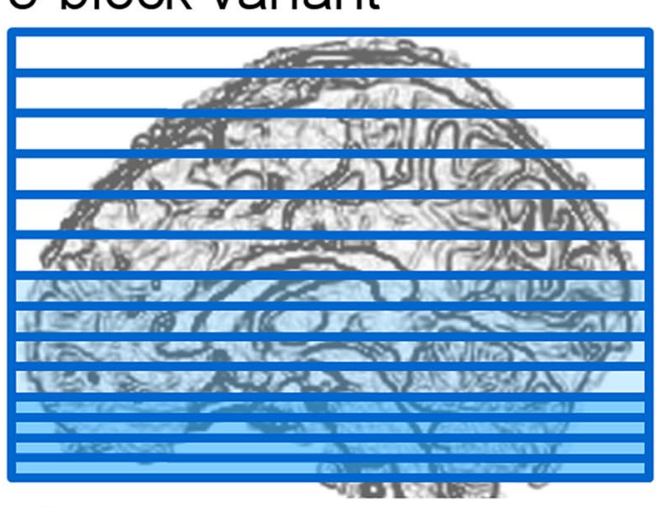

45 b

\section{2-block variant}

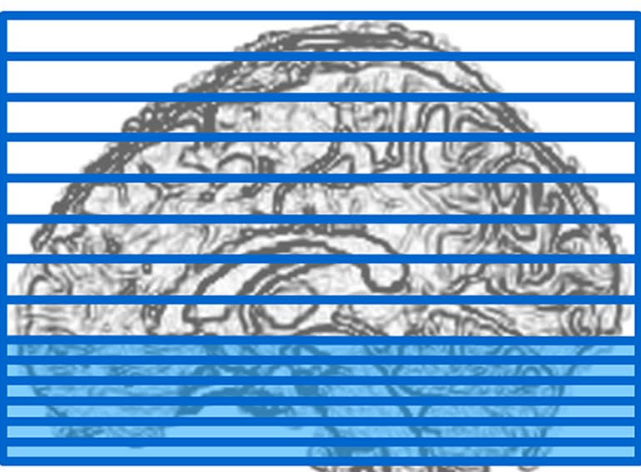

d

\section{Linear variant}

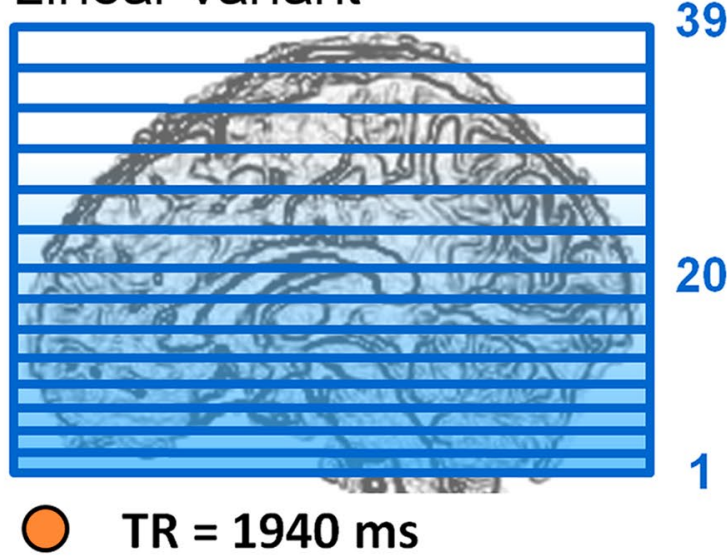

Fig. 1 Illustration of the different 2D-GE-EPI slice thickness variation schemes and approximately the same volume coverage overlaid on a normalized sagittal slice (MP-RAGE) of the 1st study. The associated volume TR is given for each method. a Control scans for a uniform slice thickness $\Delta \mathrm{z}$ of $3 \mathrm{~mm}$ (yellow) for 34 slices and $1.5 \mathrm{~mm}$ (grey) for 67 slices total with an ascending slice order from skull base to vertex. b 2-block VAST technique (green) with $\Delta \mathrm{z}=1.5 \mathrm{~mm}$ for

post hoc using Matlab 2016a (The MathWorks Inc., Sherborn, MA, USA), representing the overall voxel size of the 3-mm-thick control GE-EPI with an in-plane resolution of $1.5 \times 1.5 \mathrm{~mm}^{2}$. The (partial) higher resolution datasets of 2-block, 3-block, linear VAST, and the 1.5$\mathrm{mm}$ control were re-gridded and interpolated by cubic splines to a general voxel volume $V=6.75 \mathrm{~mm}^{3}$. Especially for susceptibility-prone brain regions, regions of interest (ROIs) were defined (Fig. 3a) and analyzed by MRIcro 1.4 (Chris Rorden, University of South Carolina, Columbia, USA; Table 2). the lower part of slice 1 to 22 and $\Delta \mathrm{z}=3 \mathrm{~mm}$ for the upper part of slice 23 to 45 . c 3-block VAST variant (blue) with $\Delta \mathrm{z}=1.5 \mathrm{~mm}$ for the lower part of slice 1 to 14 , with $\Delta \mathrm{z}=2.25 \mathrm{~mm}$ for the middle part for slice 15 to 29 and $\Delta z=3 \mathrm{~mm}$ for the upper part of slice no 30 to 45. d Linear ascending VAST method (red) with a continuous increase of $\Delta \mathrm{z}$ from slice 1 with $1.5 \mathrm{~mm}$ to slice 20 with $2.96 \mathrm{~mm}$ for the lower part and a uniform $\Delta \mathrm{z}=3 \mathrm{~mm}$ for the upper block of slice 20 to 39
For 1 subject, 150 additional volumes for each method with the same volume TR and TA were acquired without any stimulation (Fig. 3b) as resting-state fMRI [40]. Therefore, with the same GRAPPA acceleration factor [41], a separate autocalibration signal (ACS) measurement within breath-hold was conducted to prevent discontinuous tSNR results between the different techniques because of this preparation step [42]. These tSNR maps were calculated for all VAST variants, for the $3-\mathrm{mm}$ control and for the 1.5-mm control (Fig. 4) with 75 volumes, 
a

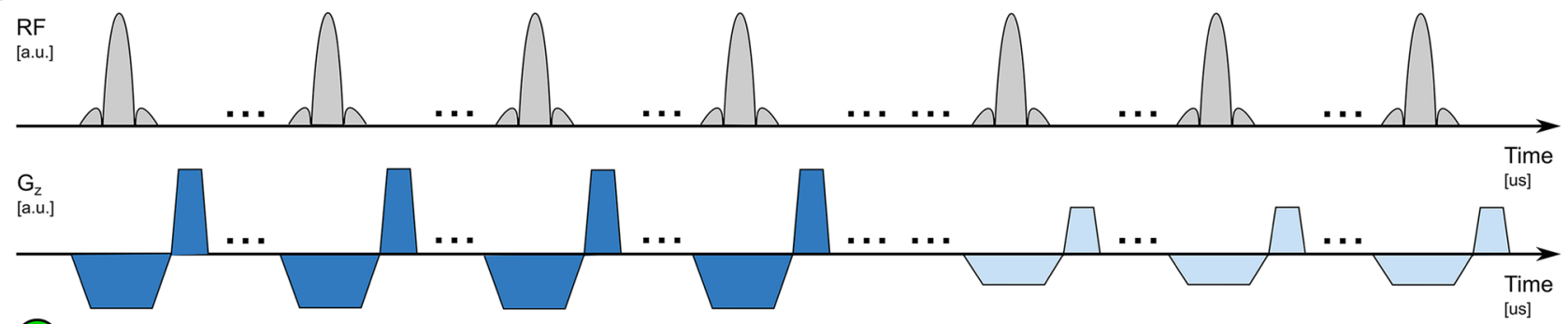

2-block varaint

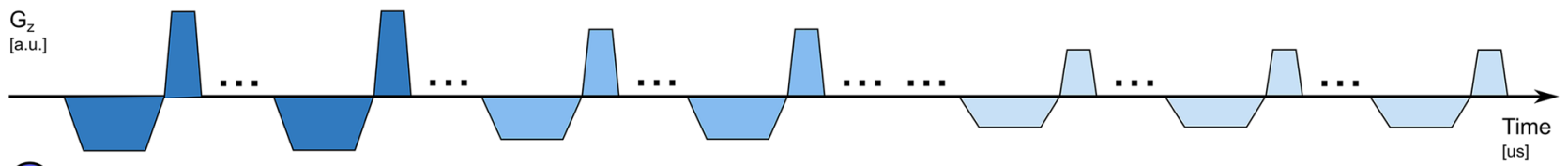

3-block varaint

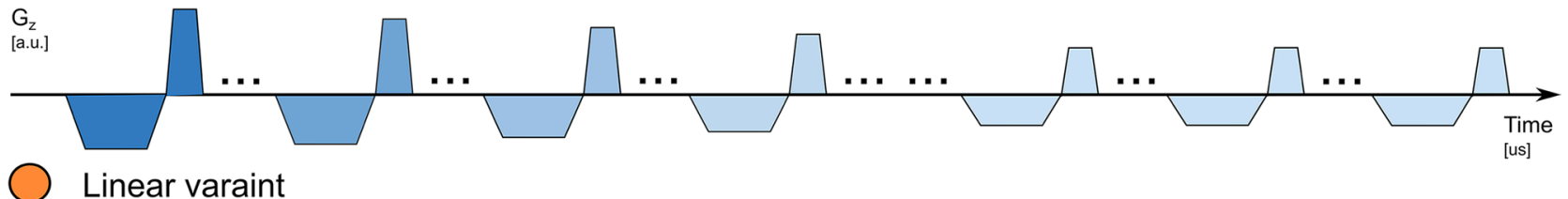

b

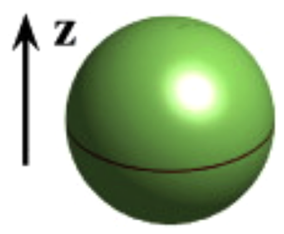

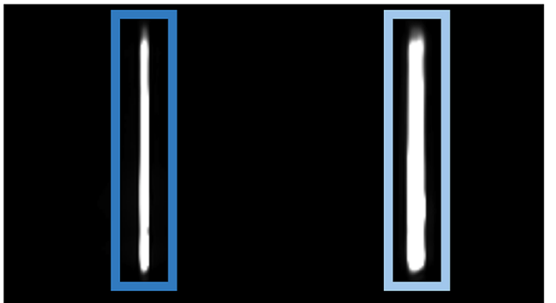

2-block varaint

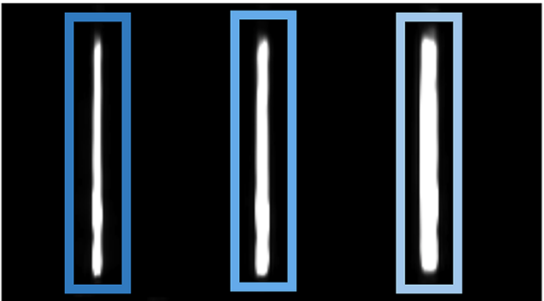

3-block varaint
Fig. 2 a Schematically pulse sequence diagrams for the three different VAST-GE-EPI slice excitation patterns. The timing and amplitudes of the slice-selection gradient $\mathrm{G}_{z}$ are depicted in conjunction with the RF sinc pulse and the subsequent rephasing gradient. In particular, the stepwise $G_{z}$ changes over time for the 2-/3-block VAST technique are highlighted and the continuous $\mathrm{G}_{z}$ amplitude reduction

a doubled TR, and halved TA (Table 1, parameters listed in brackets with dagger) with Matlab, according to Eq. 5 .

Furthermore, all datasets of the first study (as shown partly in Figs. 4 and 5), with identical whole-neocortex coverage but different volume TR/TA, were processed with Brain Voyager QX 2.8 (Brain Innovation B.V., Maastricht, The Netherlands) [43]. These represented the breath-hold fMRI measurements which are described in more detail below.

For a secondary, more practical related study, another cohort of 14 healthy volunteers ( 3 female) with a mean age of 32.6 years $(\mathrm{SD}=5.1)$ with normal or corrected to normal vision were examined with a standard finger-tapping for linear VAST can be seen. b The effect of the 2-/3-block VAST slice thickness variation is illustrated for two or three slice profiles for a circular shaped head phantom. Accordingly, the frequency encoding was chosen to be also in slice selection direction with a high distance factor

paradigm [44]. Here, normalized tSNR maps, including the cerebellum, were calculated for 2-block VAST GEEPI and the $1.5-\mathrm{mm}$ control GE-EPI from 100 volumes (Table 1, parameters listed in brackets with double dagger). These are the finger-tapping fMRI experiments which are described below.

\section{Breath-hold fMRI (1st study)}

Since the main focus of this work was the technical realization of VAST GE-EPI in comparison to normal 2D GE-EPI (Fig. 4), a robust paradigm was used for the first study to ensure a controlled global signal change within the whole-neocortex volume, as supposed by Speck et al. 


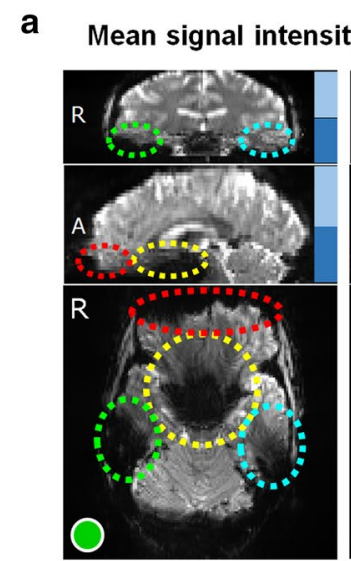

b

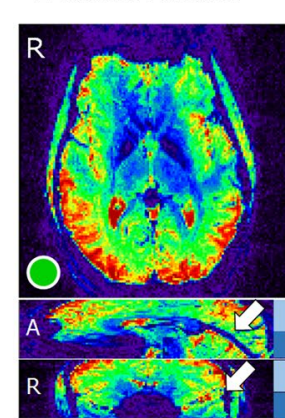

tSNR

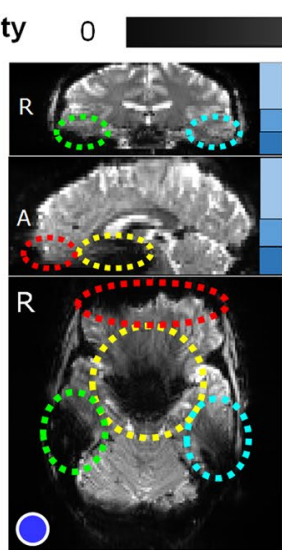

3-block variant

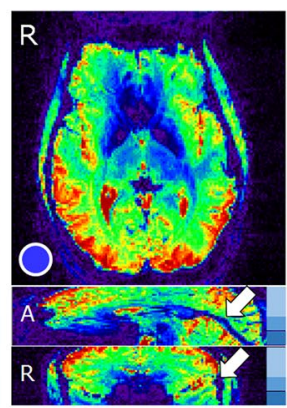

0

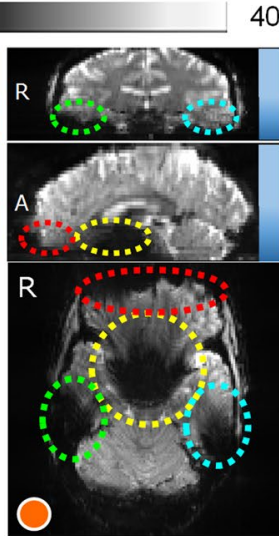

Linear variant

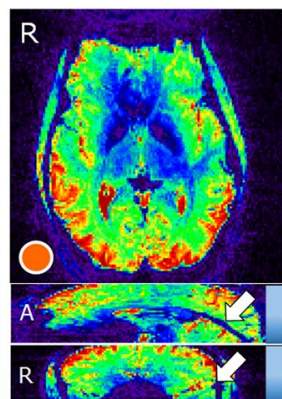

4095

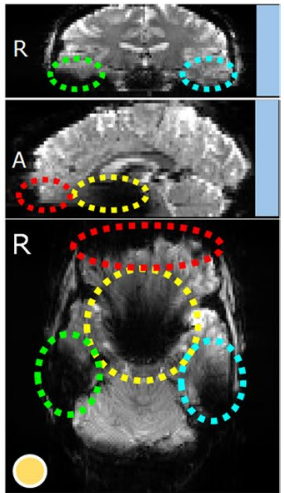

$3 \mathrm{~mm}$ control

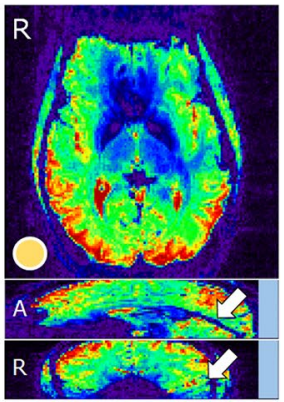

100

Fig. 3 a Depiction of the mean signal intensity distribution for the breath-hold fMRI (1st study) dataset of subject 3 on a coronal/sagittal/axial slice for all three VAST variants and the $3 \mathrm{~mm}$ control measurement after interpolation onto a general vowel size of $6.75 \mathrm{~mm}$. The extension of the different slice thicknesses $\Delta z$, according to Figure 1 with a caudal/(middle/)cranial block, is given by the bluecolored sidebars. Moreover, the four volume regions-of-interest (ROI)

[12]. Therefore, a breath-hold paradigm [45-47] was chosen, consisting of a block design with six alternating periods of $21 \mathrm{~s}$ of normal 'breathing' (green condition) and 'non-breathing' (red condition). The subjects were presented a colored full screen in accordance with both previously mentioned conditions. The last three seconds of each block consisted of a $1-\mathrm{Hz}$ change between the condition color and a black screen to prepare the subject for the next task period. Hence, the general response $[48,49]$ was modeled by convolving a canonical hemodynamic response function (HRF) with a boxcar function for 18-s duration time periods [12]. However, it should be noted that the colored screen which evoked neuronal response also includes respiration-induced signal changes, described by the respiratory response function (RRF), which is typically slower than the hemodynamic response $[50,51]$. are shown here as dotted circles for reference: The ventromedial prefrontal cortex (vmPFC) in yellow, orbitofrontal region (OFC) in red and the right/left inferior temporal gyrus (ITG) ROI in green/blue. b Time course volume signal-to-noise (tSNR) maps for an axial/sagittal/coronal slice of subject 1 before spatial interpolation. The effect of the stretched caudal parts (marked with white arrows) is visible in the sagittal/coronal slice for all VAST variants in comparison to $\mathbf{a}$

A pseudo-randomized series of the three different VAST GE-EPIs and two control GE-EPIs with $\Delta z$ of 1.5 and $3 \mathrm{~mm}$ was acquired on each subject, resulting in $5 \mathrm{fMRI}$ measurements with the identical stimulus paradigm and a total acquisition time of 4' $40^{\prime \prime}$ each. After resampling to the common voxel size $\left(6.75 \mathrm{~mm}^{3}\right)$, all datasets were realigned, coregistered with the anatomical data, and spatially normalized to Talairach space [52]. Additionally, the individual data was high-pass filtered in the temporal domain with a linear trend and a Fourier basis set of two sine/cosine functions with one/two cycles in a general linear model (GLM) approach. Furthermore, motion correction was applied using trilinear motion detection and sinc motion interpolation, as well as slice scan time correction by cubic spline interpolation. No spatial smoothing was applied for the statistical analysis on the single subject (first) level and on the multi-subject (second) level. 
Table 2 Four defined volume ROIs within the prefrontal cortex (left OFC), above the sphenoid bone (vmPFC), and within the left/right temporal lobe (left/right ITG) are listed. Refer to Figure 4 for the individual location of the spherical ROIs. For each ROI, the signal intensity mean value and standard deviation is given for the two control GE-EPI and the three different VAST-GE-EPI variants. Moreover, the relative difference to the $1.5 \mathrm{~mm}$ control mean signal intensity is stated

\begin{tabular}{|c|c|c|c|c|c|c|c|}
\hline ROI no. & Cortex region & Voxels & Liter & Variant & $\overline{\mathfrak{S}}$ & $\mathrm{SD}_{\subseteq}$ & $\begin{array}{c}\text { Rel. diff. } \\
{[\%]}\end{array}$ \\
\hline \multirow{5}{*}{1} & \multirow{5}{*}{ left OFC } & \multirow{5}{*}{5487} & \multirow{5}{*}{0.04} & $1.5 \mathrm{~mm}$ & 497.08 & 417.60 & - \\
\hline & & & & 2-block & 424.50 & 355.66 & 15 \\
\hline & & & & 3-block & 446.06 & 379.76 & 10 \\
\hline & & & & Linear & 361.92 & 283.40 & 27 \\
\hline & & & & $3 \mathrm{~mm}$ & 352.23 & 312.07 & 29 \\
\hline \multirow{5}{*}{2} & \multirow{5}{*}{ vmPFC } & \multirow{5}{*}{11588} & \multirow{5}{*}{0.08} & $1.5 \mathrm{~mm}$ & 607.60 & 327.06 & - \\
\hline & & & & 2-block & 514.80 & 257.50 & 15 \\
\hline & & & & 3-block & 530.00 & 279.00 & 13 \\
\hline & & & & Linear & 404.24 & 221.10 & 33 \\
\hline & & & & $3 \mathrm{~mm}$ & 401.92 & 251.78 & 34 \\
\hline \multirow{5}{*}{3} & \multirow{5}{*}{ left ITG } & \multirow{5}{*}{5991} & \multirow{5}{*}{0.04} & $1.5 \mathrm{~mm}$ & 403.68 & 300.68 & - \\
\hline & & & & 2-block & 335.55 & 245.27 & 17 \\
\hline & & & & 3-block & 363.64 & 270.99 & 10 \\
\hline & & & & Linear & 287.67 & 205.46 & 29 \\
\hline & & & & $3 \mathrm{~mm}$ & 274.84 & 225.53 & 32 \\
\hline \multirow{5}{*}{4} & \multirow{5}{*}{ right ITG } & \multirow{5}{*}{5836} & \multirow{5}{*}{0.04} & $1.5 \mathrm{~mm}$ & 363.76 & 307.17 & - \\
\hline & & & & 2-block & 301.89 & 249.10 & 17 \\
\hline & & & & 3-block & 327.01 & 278.67 & 10 \\
\hline & & & & Linear & 254.47 & 193.62 & 30 \\
\hline & & & & $3 \mathrm{~mm}$ & 239.67 & 211.49 & 34 \\
\hline
\end{tabular}

The statistical whole-brain data analysis on the second level was based on a random effects (RFX) GLM using a false discovery rate (FDR) [53] corrected threshold of $q<0.001$ for the contrast "breathing" versus "nonbreathing". The GLM was pre-whitened with a secondorder autoregressive method [54]. A cluster-based analysis was performed with a threshold of 100 spatially related voxels to identify compact functional groups, especially in susceptibility-prone regions. These voxel clusters were identified for the $1.5-\mathrm{mm}$ control GE-EPI with the statistically most significant voxel value $\left(t_{\text {peak }}\right)$ and its coordinates for representative brain regions in comparison to the other control and the VAST GE-EPI methods (Table 3). Moreover, for a predefined ROI within the vmPFC, the mean time course for both breathing conditions over all subjects is obtained by event-related averaging all peri-stimulus time course segments belonging to the same condition (Fig. 7).

\section{Finger-tapping fMRI (2nd study)}

Here, the 2-block VAST version's robustness is tested against the $1.5-\mathrm{mm}$ control GE-EPI in a typical fMRI experiment setup for the neocortex plus the cerebellum which involves a finger-tapping paradigm, consisting of a block design with 20 (2-block) or 14 (1.5-mm control) times alternating periods of $28 \mathrm{~s}$ tapping and rest. By randomly and sequentially tapping the different fingers of their right hand with a rate higher than one hertz, robust ipsilateral (cerebellum) and contralateral (motor cortex) neuronal activation patterns are predictable [55]. For this second study, the caudal part of the 2-block VAST GE-EPI with $\Delta z=1.5 \mathrm{~mm}$ covered most of the cerebellar volume and three dielectric bags [20] were additionally used for the posterior head region to homogenize the RF transmit field distribution for the caudal cortex/cerebellum in a typical measurement setup at $7 \mathrm{~T}$.

The lower-resolution dataset of 2-block VAST was regridded and interpolated by cubic splines to a general voxel volume $V=3.38 \mathrm{~mm}^{3}$, which equals the $1.5-\mathrm{mm}$ control measurement. tSNR maps, normalized by their volume $\sqrt{ }$ TR $[56,57]$, were calculated for both datasets and each subject after realignment, co-registration with the anatomical data, and spatial normalization on the Montreal neuroimaging (MNI) standard space [58].

\section{Results}

Figure $3 \mathrm{a}$ shows the mean signal intensity distribution for the breath-hold fMRI for all VAST GE-EPI sequences and the 3-mm control in one exemplary subject. Four different volume ROIs were defined and their mean signal intensities $(\overline{\mathfrak{S}})$ and respective SDs are reported in Table 2 for each 
Fig. 4 Qualitative comparison of the different VAST variants in subject 3 fMRI series (1st study) for zoomed views of slice 7 to slice 10 . Shown is the signal dropout for the vmPFC (Fig. 3a, yellow) and OFC (Fig. 3a, red) volume ROIs. From top to bottom, mean signal image of the $1.5-\mathrm{mm}$ control GE-EPI (grey), 2-block (green), 3-block, and linear VAST GEEPI (red), as well as the 3-mm control GE-EPI (yellow). Note that the through-plane signal dephasing effect becomes worse from top (1.5-mm control, interpolated) to bottom (3-mm control, measured)

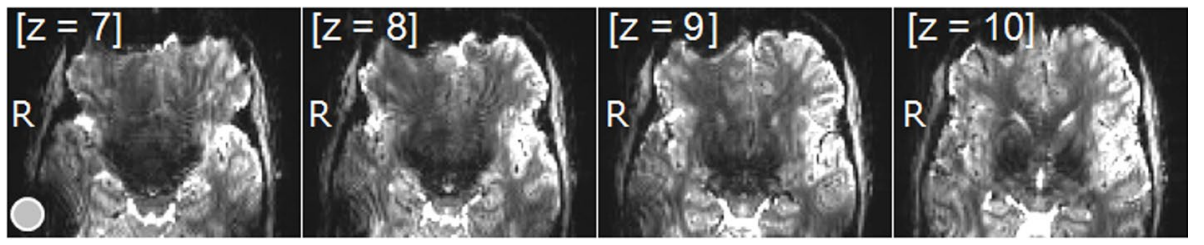

$1.5 \mathrm{~mm}$ control

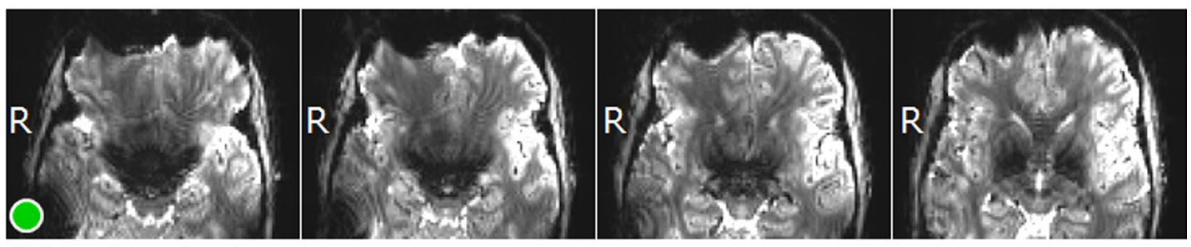

2-block variant

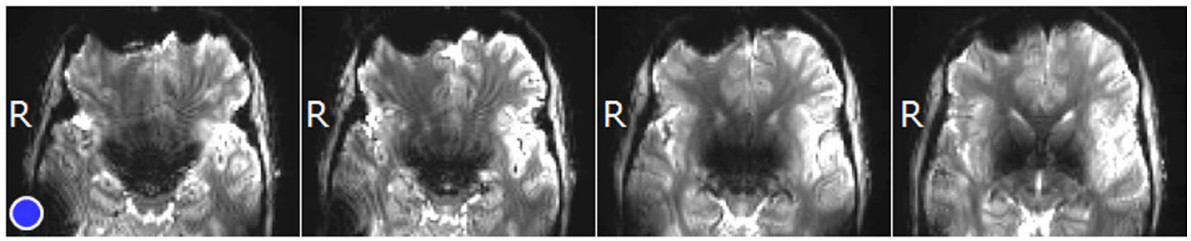

3-block variant

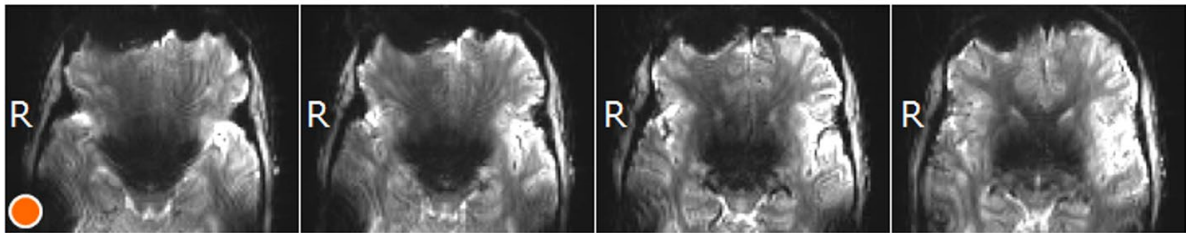

Linear variant

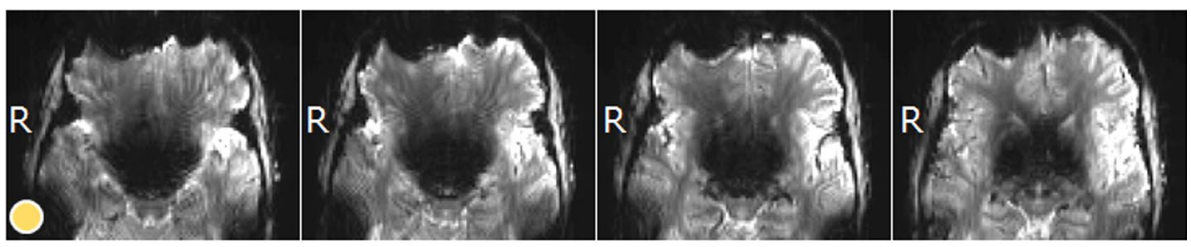

$3 \mathrm{~mm}$ control technique. From here on, the prefrontal cortex ROI near the frontal sinus (Fig. 3a, red circle) is identified as the right or left OFC ROI. The ROI above the sphenoid bone (Fig. 3a, yellow circle) is referred to as the vmPFC, plus the anterior part of the limbic and paralimbic cortex ROI. Furthermore, for this region above the sphenoid bone, the effect of the through-plane dephasing is depicted in Fig. 4. Here, the severity of signal dropouts over the different techniques can be assessed qualitatively for subject 3 (1st study). The left temporal lobe ROI near the petrous portion (Fig. 3a, blue circle) is identified as the left ITG ROI. The right temporal lobe ROI near the petrous portion (Fig. 3a, green circle) is designated as the right ITG ROI further on. For the investigated vmPFC, OFC, and ITG ROIs, the assumed basis of $\overline{\mathfrak{S}}$, given by the $1.5-\mathrm{mm}$ control measurement, is reduced by only $10 \%$ for 2-block and 3-block VAST GE-EPI in contrast to more than $30 \%$ for the control GE-EPI with 3-mm slice thickness.
Figure $3 b$ depicts the tSNR distribution of subject 1 for the three different VAST variants and the 3-mm reference scan before interpolation of the VAST GE-EPI results onto the general voxel resolution of the control scan. For a closer look on the single-subject tSNR distribution (Fig. 5), including the $1.5-\mathrm{mm}$ control, median tSNR results were calculated for the different slice thickness blocks as defined in Fig. $3 \mathrm{tSNR}_{1.5 \mathrm{~mm}}^{\text {caudal }}=(22.71 \pm 5.85)$ and $\mathrm{tSNR}_{1.5 \mathrm{~mm}}^{\text {cranial }}=(31.03 \pm 3.61)$ of the $1.5-\mathrm{mm}$ control GE-EPI, $\mathrm{tSNR}_{2 \text { block }}^{\text {caudal }}=(20.37 \pm 2.24)$ and $\mathrm{tSNR}_{2 \text { block }}^{\text {cranial }}=(39.27 \pm 3.45)$ for the 2-block variant, $\mathrm{tSNR}_{3 \text { block }}^{\text {caudal }}=(21.06 \pm 1.07), \mathrm{tSNR}_{\text {3block }}^{\text {middle }}=(33.28 \pm 4.28)$ and $\mathrm{tSNR}_{3 \text { block }}^{\text {cranial }}=(45.43 \pm 4.27)$ for the 3-block variant, tSNR $_{\text {linear }}^{\text {caudal }}=(21.66 \pm 5.26)$ and $\mathrm{tSNR}_{\text {linear }}^{\text {cranial }}=(31.42 \pm 2.78)$ for the linear variant of VAST GE-EPI, and $\mathrm{tSNR}_{3 \mathrm{~mm}}^{\text {caudal }}=(29.18 \pm 5.92)$ and $\mathrm{tSNR}_{3 \mathrm{~mm}}^{\text {cranial }}=(44.2 \pm 3.19)$ for the 3 -mm control GE-EPI. Note that the caudal median tSNR values of all VAST 
$\mathrm{R}$

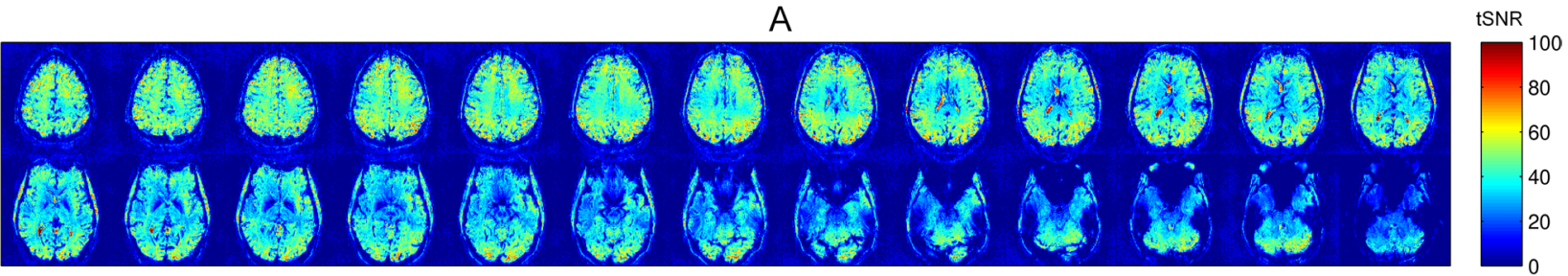

$1.5 \mathrm{~mm}$ control

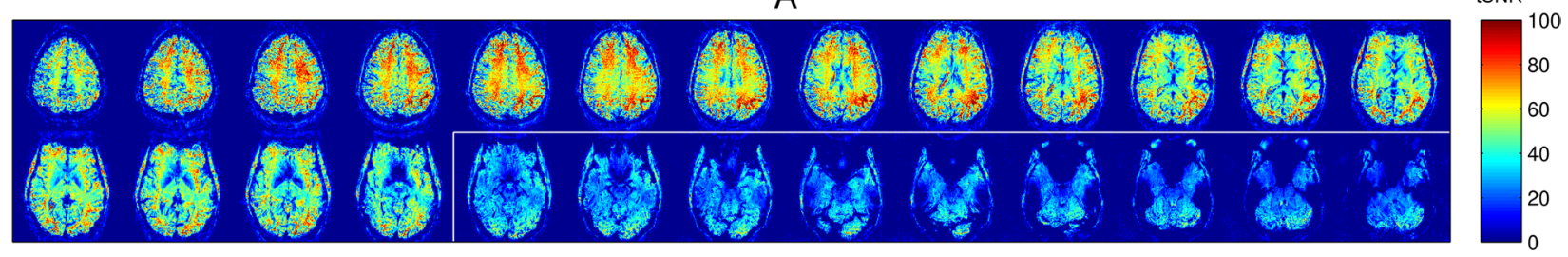

2-block variant

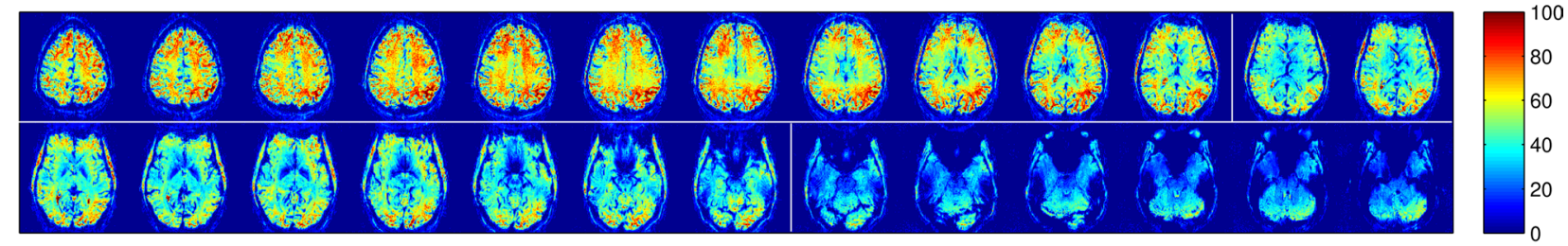

3-block variant

A

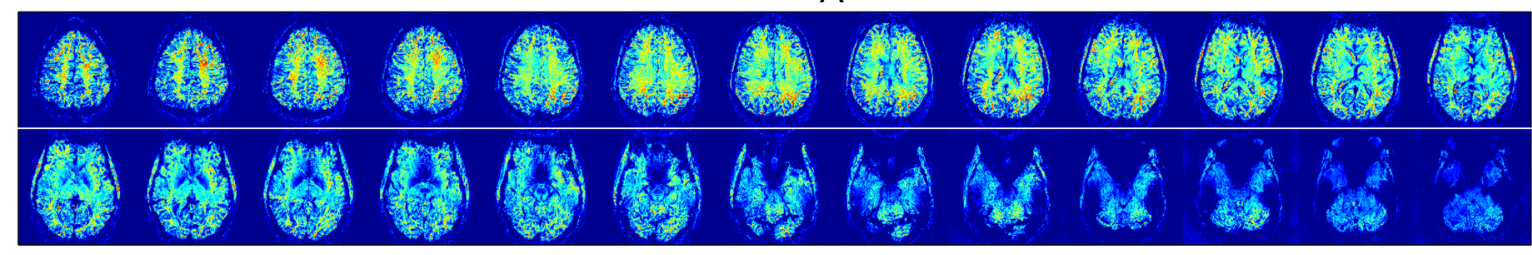

Linear variant

$\mathrm{R}$

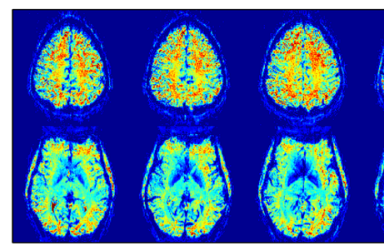

$3 \mathrm{~mm}$ control

Fig. 5 Single-subject tSNR results of the 1st study (subject 1) for all VAST variants and both control measurements, at equal TR but different volume coverage. VAST data were intensity-corrected and interpolated onto a common voxel size corresponding to the 3-mm control. As expected, the values for the interpolated 1.5-mm control measurement are lower. We observed that the between-technique variation of the VAST variants was strongly affected by the quality of the GRAPPA ACS acquisition at the beginning of each run, likely due to

variants versus the $1.5-\mathrm{mm}$ control measurement and the cranial median tSNR values of 2/3-block VAST versus 3-mm control do not differ much. In general, due to the different $\sigma_{\text {ratio }}$ values for the variable $\Delta z$ of the VAST variants, the tSNR per slice varies in accordance to the control measurements with $\Delta z=1.5 \mathrm{~mm}$ for the caudal
A

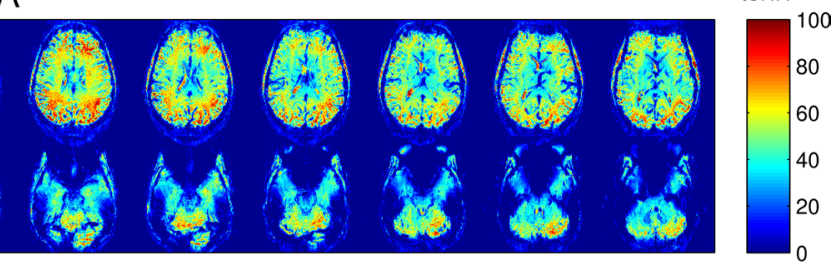

tSNR

100

tSNR

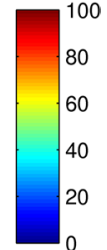


Table 3 ROI analysis results of four representative statistically significant voxel clusters with a minimum cluster size of 100 voxels $(\hat{=}$ $675 \mathrm{~mm}^{3}$ ) within the frontal and temporal cortex. The voxel cluster peak t-values of the RFX GLM with $\mathrm{q}<0.001$ (FDR corrected) in conjunction with their respective Talairach coordinates and the attrib- uted Brodmann area (BA) are stated. Note that for the $3 \mathrm{~mm}$ control GE-EPI method (yellow rows) no statistical significant voxels within the left ACC or the right ITG were detectable, in contrast to the left $\mathrm{OFC}$ and right parahippocampal gyrus

\begin{tabular}{|c|c|c|c|c|c|c|}
\hline \multirow[t]{2}{*}{ Cluster no. } & \multirow[t]{2}{*}{ Cortex region } & \multirow[t]{2}{*}{ BA } & Variant & Voxels & $t_{\text {peak }}$ & {$[x / y / z]_{\text {Talairach }}$} \\
\hline & & & $1.5 \mathrm{~mm}$ & 118 & 6.71 & $-1 / 49 /-10$ \\
\hline \multirow{4}{*}{1} & \multirow{4}{*}{ left OFC } & \multirow{4}{*}{11} & 2-block & 362 & 7.77 & $-3 / 44 /-6$ \\
\hline & & & 3-block & 435 & 9.54 & $-1 / 53 /-6$ \\
\hline & & & Linear & 436 & 7.28 & $-7 / 46 /-9$ \\
\hline & & & $3 \mathrm{~mm}$ & 213 & 9.3 & $0 / 46 /-9$ \\
\hline \multirow{5}{*}{2} & \multirow{5}{*}{ left ACC } & \multirow{5}{*}{24} & $1.5 \mathrm{~mm}$ & 479 & 8.24 & $-4 / 21 /-6$ \\
\hline & & & 2-block & 369 & 7.63 & $-1 / 14 /-9$ \\
\hline & & & 3-block & 460 & 8.59 & $-1 / 19 /-9$ \\
\hline & & & Linear & 960 & 10.33 & $-4 / 22 /-3$ \\
\hline & & & $3 \mathrm{~mm}$ & - & - & - \\
\hline \multirow{5}{*}{3} & \multirow{5}{*}{$\begin{array}{c}\text { right } \\
\text { Parahippocampal } \\
\text { gyrus }\end{array}$} & \multirow{5}{*}{ - } & $1.5 \mathrm{~mm}$ & 400 & 8.19 & $21 /-2 /-9$ \\
\hline & & & 2-block & 629 & 8.57 & $20 /-2 /-9$ \\
\hline & & & 3-block & 613 & 10.36 & $23 /-2 /-9$ \\
\hline & & & Linear & 495 & 10.43 & $23 /-2 /-9$ \\
\hline & & & $3 \mathrm{~mm}$ & 387 & 9.51 & $23 /-2 /-12$ \\
\hline \multirow{5}{*}{4} & \multirow{5}{*}{ right ITG } & \multirow{5}{*}{20} & $1.5 \mathrm{~mm}$ & 188 & 7.08 & $41 /-32 /-18$ \\
\hline & & & 2-block & 648 & 10.49 & $41 /-35 /-18$ \\
\hline & & & 3-block & 563 & 9.15 & $44 /-35 /-15$ \\
\hline & & & Linear & 740 & 8.57 & $36 /-37 /-23$ \\
\hline & & & $3 \mathrm{~mm}$ & - & - & - \\
\hline
\end{tabular}

A

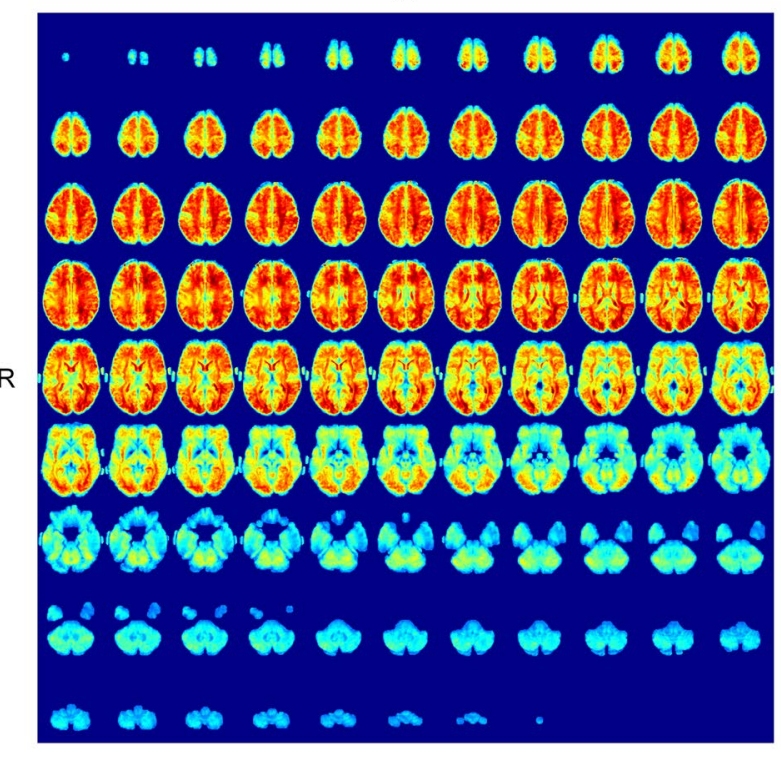

$1.5 \mathrm{~mm}$ control

Fig. 6 Group tSNR maps of the 2nd study for the 1.5-mm control GE-EPI (left) and 2-block VAST GE-EPI (right). SNR was normalized by $\sqrt{T R}$ for comparison of the protocols in terms of tSNR per unit time. Different total slice count and, hence, TR were employed to
A

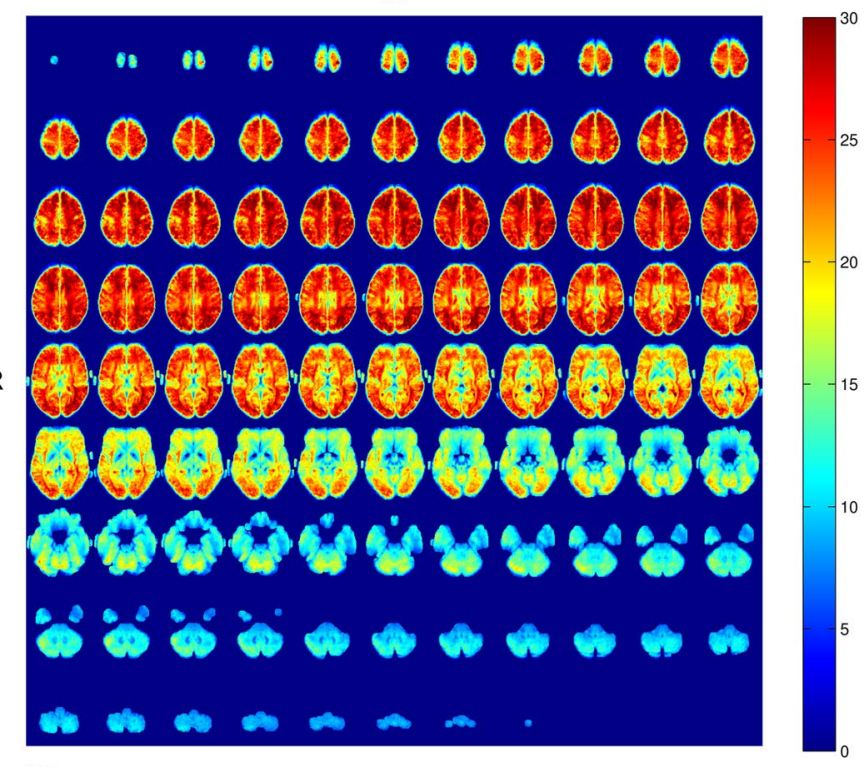

2-block variant

achieve the same volume coverage. The caudal slices both show good consistency while the 2-block variant slices, with a doubled slice thickness that extends over $2 / 3$ of the volume, provide higher values in accordance with the single-subject results of Fig. 5 


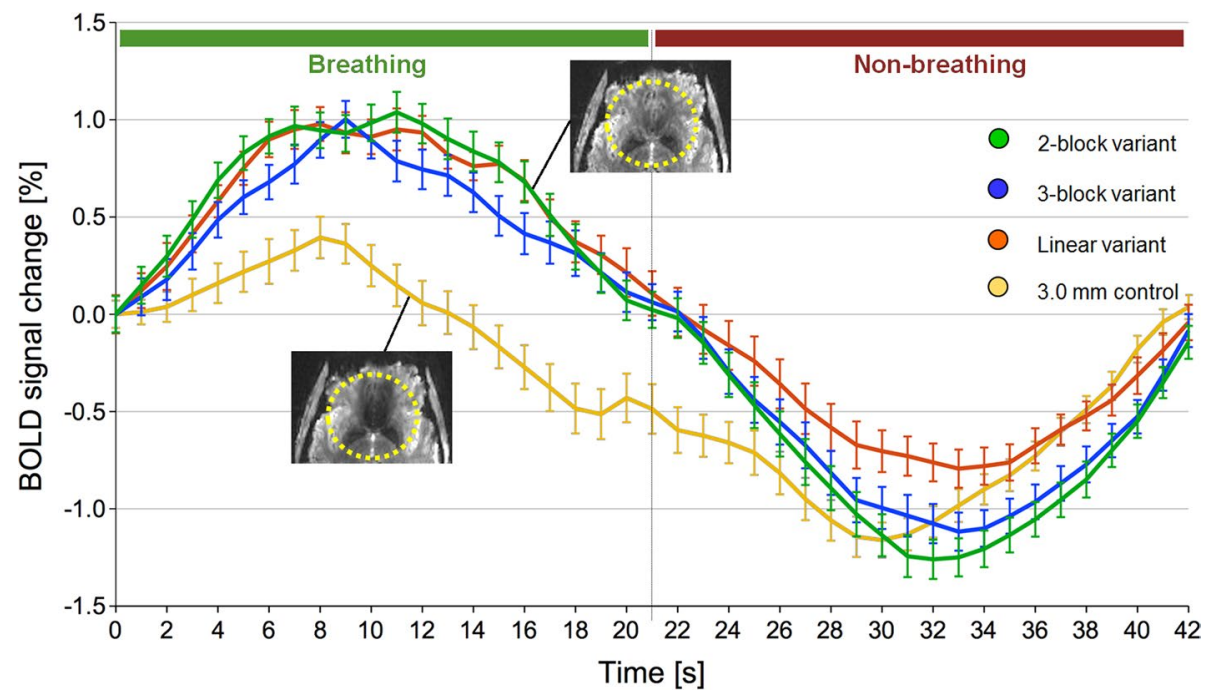

Fig. 7 Event-related averaged BOLD percent signal changes, including the respiratory-variation-related fluctuations, over all condition blocks and all subjects $(N=12)$ for the 1st study are shown. These are located within the vmPFC volume ROI above the sphenoid bone for the 3-mm control GE-EPI (yellow) measurement and the interpo- lated 2-block (green), 3-block (blue), and linear VAST GE-EPI (red) techniques. The error bars per time step (volume) indicate the standard deviation for the subject cohort. As in Fig. 3, the vmPFC ROI is depicted (yellow circle) for 2-block VAST GE-EPI and 3-mm control GE-EPI of subject 3

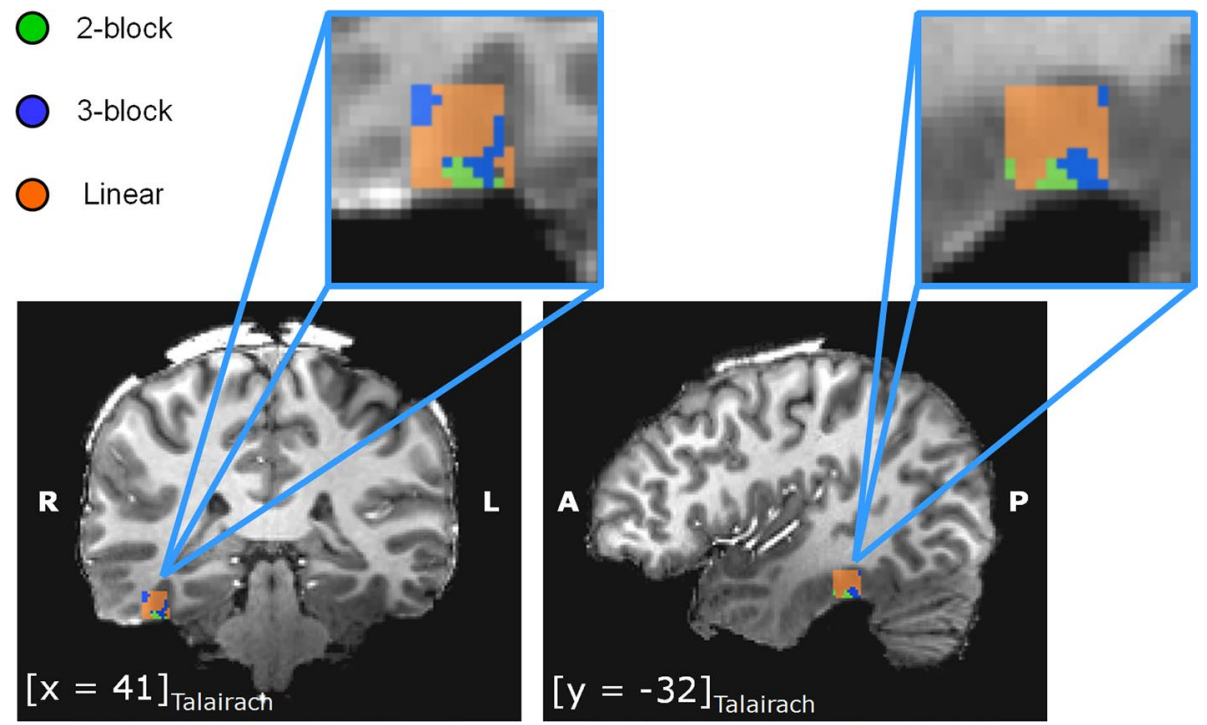

Fig. 8 The extension of statistically significant group activation patterns $(N=12)$ of voxel cluster 4 is depicted for the 1 st study, listed within Table 2 for the right ITG. The underlying contrast is based on a multi-subject RFX GLM with $q<0.001$ (FDR-corrected). The three different methods overlap each other and are listed from top to bottom while the aforementioned include the following voxel-wise

these notable between-measurement variations are due to increased physiological noise on the single-subject level, especially for the linear VAST variant, which can be omitted by a group tSNR analysis that is shown for the $1.5-\mathrm{mm}$ control GE-EPI and 2-block VAST GE-EPI (2nd study) in 2-block (green), 3-block (blue), and linear VAST GE-EPI (red). The increased caudal spatial extension of the 2-/3-block VAST variant's significant voxel cluster next to the petrous part of the temporal bone show that these techniques are less prone to susceptibility artifacts for this cortex region. Note that for the 3-mm control GE-EPI technique, no statistically significant voxels are visible at all

Fig. 6. Here, the cranial slices ( $2 / 3$ of the whole volume) of the 2-block VAST variant, measured with $\Delta z=3 \mathrm{~mm}$ and interpolated onto $1.5 \mathrm{~mm}$, provide $1.2-1.6$ times higher median tSNR values in accordance with the single-subject results of Fig. 5. 
Moreover, these brain regions were investigated in a hypothesis-driven approach by event-related averaging over all 'breathing' and 'non-breathing' conditions for the complete subject cohort of one exemplar ROI in Fig. 8. For this vmPFC volume ROI over all subjects, the averaged absolute maximum/minimum BOLD percent signals, its standard errors, and the resulting percent points (pp) range between these two values for the different techniques are $\mathrm{BOLD}_{2-\text { block }}^{\min / \max }=(-1.26 / 1.04 \pm 0.1 / 0.1) \%$ with $\mathrm{BOLD}_{2-\text { block }}^{\text {range }}=2.3 \mathrm{pp}$ (Fig. 7, green line), $\mathrm{BOLD}_{3-\text { block }}^{\mathrm{min} / \mathrm{max}}$ $=(-1.12 / 1.0 \pm 0.1 / 0.11) \%$ with BOLD $_{3-\text { block }}^{\text {range }}=2.12 \mathrm{pp}$ (Fig. 7, blue line), BOLD $\operatorname{minear}_{\text {min }}^{\max }=(-0.79 / 0.98 \pm 0$. 08/0.11)\% with BOLD $_{\text {linear }}^{\text {range }}=1.77$ pp (Fig. 7, red line), and $\mathrm{BOLD}_{3 \mathrm{~mm}}^{\mathrm{min} / \mathrm{max}}=(-1.16 / 0.39 \pm 0.09 / 0.16) \%$ with $\mathrm{BOLD}_{3 \mathrm{~mm}}^{\text {range }}=1.55 \mathrm{pp}$ (Fig. 7, yellow line).

Additionally, four dedicated voxel clusters were identified for the second-level analysis, which showed a statistically significant contrast for the main effect of almost all techniques in Table 3 . For cluster 1 , representing the left OFC, the 3-block and linear variants have both the largest coverage with 435 and 436 voxels, but the 3-block VAST GE-EPI had the highest value, with $t_{\text {peak }}=9.54$. On the one hand, the left anterior cingulate cortex (ACC) within cluster 2 showed no significant voxels for the 3-mm control GE-EPI at $q<0.001$ (FDR-corrected). On the other hand, the linear variant expanded over 960 total voxels with $t_{\text {peak }}=10.33$. Cluster 3 , within the right parahippocampal gyrus, with 629 voxels and 613 voxels had the largest size for the 2-block and the 3-block variant, while here, $t_{\text {peak }}=10.36$ for 3 -block VAST GE-EPI and $t_{\text {peak }}=10.43$ for linear VAST GE-EPI. The right ITG (cluster 4) maximum size was 740 voxels for the linear variant, while the maximum $t_{\text {peak }}=10.49$ for 2-block VAST GE-EPI, while also here, no statistical significant voxel could be registered for the 3-mm control measurement. For conclusion of the above cluster findings, both block techniques of the VAST GE-EPI sequence show the widest cluster extension which can be seen exemplarily for cluster 4 in Fig. 8 .

The spatial extent of the latter cluster is demonstrated on the co-registered and normalized high-resolution anatomical dataset of one subject. Figure 8 shows the farthest caudal extent of voxel cluster 4 statistically significant activation patterns, closest to the petrous portion, for the 2-block and 3-block variant on an example coronal and sagittal slice.

\section{Discussion}

The proposed VAST GE-EPI technique was implemented in three different ways for examination of its utility for functional whole-brain imaging with reduced susceptibility artifact signal losses and a higher temporal sampling rate. Therefore, the general signal distribution and the tSNR aspects on the individual subject level were analyzed before the sequence performances for multi-subject functional imaging were considered. Moreover, a group analysis on normalized tSNR distribution was performed for 2-block VAST and the 1.5-mm control measurement to compensate for the between-scan data variability of one single subject.

As expected and qualitatively shown in Figs. 3 and 4, the through-plane signal dephasing near air-filled cavities could be reduced by the use of thinner slices [29]. Although the susceptibility-induced artifact could be reduced, it has been shown previously that this improvement comes at the expense of an overall reduced SNR [29].

In Fig. 5, only one example subject result for the mean signal intensity and tSNR distribution of the first study is presented. The findings are, to a large extent, consistent with the physiological-to-thermal noise ratios reported in the literature [32, 33] and its effects on the tSNR distribution. The single-subject tSNR variability for the datasets with GRAPPA acceleration factor $R=3$ have to be discussed under the scope of the separate ACS acquisition at the beginning of each sequence [59, 60]. Especially for the caudal brain regions, the voxel volumes were chosen so that $\sigma_{\text {ratio }}=1$, as it has been previously suggested for lower magnetic field strength [61]. Furthermore, spatial smoothing by interpolation of the data leads to comparable results with the control GE-EPI data without adding physiological noise [32, 33]. Moreover, the normalized group tSNR results of Fig. 6 are consistent with this statement and provide a more robust overview of the 2-block VAST GE-EPI performance in comparison to the $1.5-\mathrm{mm}$ control measurement.

The full potential of VAST GE-EPI is exploited especially for a breath-hold fMRI paradigm, which allows for robust non-specific brain region task-related statistical contrast with less sensitivity to habituation effects or interscan motion artifacts [45]. For the vmPFC singlesubject volume ROI, the findings resulted into a 54-61\% increased time-course difference between the maximum and minimum BOLD values. This is intriguing, since this region is located near the limbic system with the considered substructures of the amygdalae [62]. Together with the prefrontal cortex region these regions take part in several higher human cognitive processes and are subject to intensive research $[63,64]$. The multi-subject vmPFC ROI event-related averaging analysis reaffirmed the results with a 48\% (2-block), 37\% (3-block), and 14\% (linear) increased BOLD signal change for VAST GE-EPI in comparison to the 3-mm control GE-EPI.

Depending on the anatomical location and the coverage with thin slice selection profiles, several maximum 
$t_{\text {peak }}$ values for the OFC, ACC, parahippocampal gyrus, and ITG region could be reported, while for the 3-mm control technique, no statistically significant cluster could be reported for the ACC and ITG. Due to the nature of the breath-hold paradigm, the statistical analysis was conducted with a rather strict thresholding. Therefore, the statistically significant cluster expansion of the different VAST contrasts could be illustrated for the grey matter near the air-filled cavities of the sphenoid bone and the right petrous portion. Furthermore, it has been shown that the investigated Brodmann's areas 11, 20, and 24 have comparable mean percent signal changes of $\sim 2.4-3 \%$ and a mean amount of statistically significant active voxels with $\sim 0.16-0.2$ during a breath-hold fMRI exam at $3 \mathrm{~T}$ [49]. Apart from its anatomical location near air-filled cavities, the area's comparability in vascularization by a functional analysis of their $t_{\text {peak }}$ values is given rather than it is for other regions, like the motor or the visual cortex.

Of the whole-neocortex breath-hold fMRI variants investigated here, the implementation of the 2-block VAST GE-EPI proved to be the best alternative to the susceptibility-prone 3-mm control GE-EPI with a faster sampling rate. As for the much slower sampled 1.5-mm control GE-EPI, all of the brain region's signal covered by the thinner caudal slice part of the 2-block VAST GE-EPI was preserved. The three different VAST GE-EPI variants were implemented to show proof-of-concept. Within the scope of this tailored examination, we found that the linearly varying slice thickness implementation is less suited for the anatomical circumstances than the static block-wise slice thickness variation. For further improvement of the VAST technique, a flexible scheme for slice thickness variation could be determined based on an individual B0 field map pre-scan [34].

The BOLD activation paradigm of the first study was chosen for its robustness to produce overall statistical significant active voxels, showing proof-of-concept for the VAST technique. For evaluation of the potentials of VAST GE-EPI, dedicated fMRI paradigms with specific neurological research questions of (fast) event-related design would be the next logical step. The group-normalized tSNR maps for one VAST technique in contrast to the control GE-EPI proved the applicability of variable slice thicknesses for a standard finger-tapping paradigm of the second study. Although the different noise characteristics have to be considered for a whole-brain functional analysis, 2-block VAST GE-EPI provided the same high-resolution wholebrain coverage results without signal dropout than the control, but within only two thirds of the originally required time. Therefore, it has been shown for the whole brain, without tailoring the analysis for caudal ROIs only, that the VAST technique with a higher sampling rate is as effective as the much slower control measurement.
2D (VAST) GE-EPI methods provide possibilities for dedicated slice-by-slice B0 and/or RF-shimmed singleor multi-slice excitation [26, 65]. Because of the nonequidistant slices through the volume, the VAST GE-EPI schemes cannot easily be combined with multi-band excitations as in 2D SMS-EPI [66]. This fact is a clear limitation when it comes to time-optimized and highly accelerated imaging procedures, such as the ones currently being used for the Human Connectome Project [67]. Moreover, the current developments of 3D EPI in combination with CAIPI(RINHA) allow for fast sub-millimeter isometric voxel sampling without severe susceptibility artifacts [68-70]. For future work, a comparison between 3D EPICAIPI, 2D SMS-EPI, and 2D VAST EPI should be conducted, while many aspects of the different techniques have to be considered and each method has its merits and drawbacks.

Several studies on 2D GE-EPI with slice-dependent parameter variations exist, which, for instance, apply a different TE [17], vary the slice-selection refocussing gradient strength [39], or combine GE-EPI with spin echo imaging for the inferior part [71]. While, in general, these methods conceptually bear some similarity to the VAST EPI method proposed here, the approach of using slice position-dependent thickness adjustment has not been explored previously, to the best of our knowledge.

This pragmatic approach to counter susceptibility artifacts offers some new possibilities for slice-specific RF shimming with reduced signal voids by adaption to the local anatomical conditions within a reasonable measurement time. Recently, promising progress for UHF head slice-by-slice RF shimming [72] and spoke pulse design application within strong B0 field variations [26] has been made. By combining the best of both worlds for compensation of the transmit RF field variations on one hand and, on the other hand, for removal of the static B0 field susceptibility artifacts, the VAST GE-EPI method can be improved further. Especially for the cerebellum, the benefit by implementation of VAST in combination with a semi-dynamic RF shimming procedure for a dedicated head-neck RF coil combination was currently shown [73].

\section{Conclusion}

This work for $7 \mathrm{~T}$ UHF MRI introduces the 2D EPI acquisition strategy termed VAST, in which the slice thickness is varied over the imaging volume. Thinner slices reduce the signal voids by susceptibility artifacts in inferior brain regions, while thicker slices efficiently sample unproblematic superior brain regions, keeping whole-brain TR manageable. This provides a method for whole-brain GE-EPI with less artifacts and increased flexibility for a 
whole-brain fMRI imaging protocol. The implementation is easy, the slice thickness variation can be setup to match the individual anatomical situation, and it can be combined with other methods for in vivo main magnetic field and transmit field homogenization.

Authors' contribution S Brunheim: project development, programming, data collection, data analysis, manuscript writing. S Johst: project development, programming, data collection, manuscript editing. V Pfaffenrot: data collection, data analysis, manuscript editing. S Maderwald: data collection, manuscript editing. HH Quick: project supervision, manuscript editing. BA Poser: project supervision, project development, programming, data analysis, manuscript editing.

\section{Compliance with ethical standards}

Conflict of interest The authors declare that they have no conflicts of interest.

Ethical approval All procedures performed in studies involving human participants were in accordance with the ethical standards of the institutional and/or national research committee and with the 1964 Helsinki Declaration and its later amendments or comparable ethical standards.

Informed consent Informed consent was obtained from all individual participants included in the study.

\section{References}

1. Pauling L, Coryell CD (1936) The magnetic properties and structure oh hemoglobin, oxyhemoglobin and carbonmonoxyhemoglobin. Proc Natl Acad Sci USA 22:210-216

2. Norris DG (2006) Principles of magnetic resonance assessment of brain function. J Magn Reson Imaging 23:794-807

3. Ogawa S, Menon RS, Tank DW, Kim S-G, Merkle H, Ellermann JM, Ugurbil K (1993) Functional brain mapping by blood oxygenation level-dependent contrast magnetic resonance imaging. Biophys J 64:803

4. van der Zwaag W, Francis S, Head K, Peters A, Gowland P, Morris P, Bowtell $\mathrm{R}$ (2009) fMRI at 1.5, 3 and $7 \mathrm{~T}$ : characterising BOLD signal changes. Neuroimage 47:1425-1434

5. Constable RT (1995) Functional MR imaging using gradientecho echo-planar imaging in the presence of large static field inhomogeneities. J Magn Reson Imaging 5:746-752

6. Ojemann JG, Raichle ME, Conturo TE (1997) Anatomic localization and quantitative analysis of gradient refocused echo-planar fMRI susceptibility artifacts. Neuroimage 167:156-167

7. Jezzard P, Clare S (1999) Sources of distortion in functional MRI data. Hum Brain Mapp 8:80-85

8. Jezzard P (2012) Correction of geometric distortion in fMRI data. Neuroimage 62:648-651

9. Deichmann R, Josephs O, Hutton C, Corfield DR, Turner R (2002) Compensation of susceptibility-induced BOLD sensitivity losses in echo-planar fMRI imaging. Neuroimage 15:120-135

10. Mansfield P, Maudsley AA (1977) Planar spin imaging by NMR. J Magn Reson 27:101-119

11. Weiskopf N, Hutton C, Josephs O, Turner R, Deichmann R (2007) Optimized EPI for fMRI studies of the orbitofrontal cortex: compensation of susceptibility-induced gradients in the readout direction. Magn Reson Mater Phy 20:39-49
12. Speck Oliver, Stadler J, Zaitsev M (2008) High resolution singleshot EPI at 7T. Magn Reson Mater Phy 21:73-86

13. Robinson S, Windischberger C, Rauscher A, Moser E (2004) Optimized 3 T EPI of the amygdalae. Neuroimage 22:203-210

14. Glover GH (1999) 3D z-Shim method for reduction of susceptibility effects in BOLD fMRI. Magn Reson Med 42:290-299

15. Weiskopf N, Hutton C, Josephs O, Deichmann R, Gottfried JA, Hutton C, Turner R, Weiskopf N, Hutton C, Josephs O, Deichmann R (2006) Optimal EPI parameters for reduction of susceptibility-induced BOLD sensitivity losses: a whole-brain analysis at $3 \mathrm{~T}$ and $1.5 \mathrm{~T}$. Neuroimage 33:493-504

16. Frahm J, Merboldt K-D, Hänicke W (1988) Direct FLASH $\{\mathrm{MR}\}$ imaging of magnetic field inhomogeneities by gradient compensation. Magn Reson Med 6:474-480

17. Stöcker T, Kellermann T, Schneider F, Habel U, Amunts K, Pieperhoff P, Zilles K, Shah NJ (2006) Dependence of amygdala activation on echo time: results from olfactory fMRI experiments. Neuroimage 30:151-159

18. Cusack R, Russell B, Cox SML, De Panfilis C, Schwarzbauer C, Ansorge R (2005) An evaluation of the use of passive shimming to improve frontal sensitivity in fMRI. Neuroimage 24:82-91

19. Yang QX, Mao W, Wang J, Smith MB, Lei H, Zhang X, Ugurbil K, Chen W (2006) Manipulation of image intensity distribution at 7.0 T: passive RF shimming and focusing with dielectric materials. J Magn Reson Imaging 24:197-202

20. Teeuwisse WM, Brink WM, Haines KN, Webb AG (2012) Simulations of high permittivity materials for $7 \mathrm{~T}$ neuroimaging and evaluation of a new barium titanate-based dielectric. Magn Reson Med 67:912-918

21. Cho ZH, Ro YM (1992) Reduction of susceptibility artifact in gradient-echo imaging. Magn Reson Med 23:193-200

22. Stenger VA, Boada FE, Noll DC (2000) Three-dimensional tailored RF pulses for the reduction of susceptibility artifacts in $\mathrm{T} * 2$-weighted functional MRI. Magn Reson Med 44:525-531

23. Yang C, Deng W, Alagappan V, Wald LL, Stenger VA (2010) Four-dimensional spectral-spatial RF pulses for simultaneous correction of B1 + inhomogeneity and susceptibility artifacts in T 2*-weighted MRI. Magn Reson Med 64:1-8

24. Deng W, Yang C, Alagappan V, Wald LL, Boada FE, Stenger VA (2009) Simultaneous Z-shim method for reducing susceptibility artifacts with multiple transmitters. Magn Reson Med 61:255-259

25. Schneider R, Boada F, Haueisen J, Pfeuffer J (2015) Automated slice-specific simultaneous $\mathrm{Z}$-shim method for reducing B1 inhomogeneity and susceptibility-induced signal loss with parallel transmission at 3T. Magn Reson Med 74:934-944

26. Guérin B, Stockmann JP, Baboli M, Torrado-Carvajal A, Stenger AV, Wald LL (2016) Robust time-shifted spoke pulse design in the presence of large B0 variations with simultaneous reduction of through-plane dephasing, B1+ effects, and the specific absorption rate using parallel transmission. Magn Reson Med 76:540-554

27. Li TQ, van Gelderen P, Merkle H, Talagala L, Koretsky AP, Duyn J (2006) Extensive heterogeneity in white matter intensity in high-resolution $\mathrm{T} 2 *$-weighted MRI of the human brain at 7.0 T. Neuroimage 32:1032-1040

28. Van de Moortele PF, Auerbach EJ, Olman C, Yacoub E, Uğurbil K, Moeller S (2009) T1 weighted brain images at 7 Tesla unbiased for proton density, $\mathrm{T} 2 *$ contrast and $\mathrm{RF}$ coil receive $\mathrm{B} 1$ sensitivity with simultaneous vessel visualization. Neuroimage 46:432-446

29. Merboldt K-D, Finsterbusch J, Frahm J (2000) Reducing inhomogeneity artifacts in functional MRI of human brain activation-thin sections vs gradient compensation. J Magn Reson 145:184-191 
30. Krüger G, Glover GH (2001) Physiological noise in oxygenation-sensitive magnetic resonance imaging. Magn Reson Med 46:631-637

31. Triantafyllou C, Krueger G, Wiggins CJ, Potthast A, Wiggins GC, Wald LL (2005) Comparison of physiological noise at 1.5 $\mathrm{T}, 3 \mathrm{~T}$ and $7 \mathrm{~T}$ and optimization of fMRI acquisition parameters. Neuroimage 26:243-250

32. Triantafyllou C, Hoge RD, Wald LL (2006) Effect of spatial smoothing on physiological noise in high-resolution fMRI. Neuroimage 32:551-557

33. Murphy K, Bodurka J, Bandettini PA (2007) How long to scan? The relationship between fMRI temporal signal to noise ratio and necessary scan duration. Neuroimage 34:565-574

34. Gruetter R, Tkáč I (1993) Automatic, localized in vivo adjustment of all first- and second-order shim coils. Magn Reson Med 29:804-811

35. Fautz H, Vogel M, Gross P (2008) B1 mapping of coil arrays for parallel transmission. Intl Soc Mag Reson Med, Proc, $p$ 1247

36. Mugler JP, Brookeman JR (1990) Three-dimensional magnetization-prepared rapid gradient-echo imaging (3D MP RAGE). Magn Reson Med 15:152-157

37. Kupce E, Freeman R (1995) Adiabatic pulses for wideband inversion and broadband decoupling. J Magn Reson Ser A 115:273-276

38. Wrede KH, Johst S, Dammann P, Umutlu L, Schlamann MU, Sandalcioglu IE, Sure U, Ladd ME, Maderwald S (2012) Caudal image contrast inversion in MPRAGE at 7 Tesla. Problem and Solution. Acad Radiol 19:172-178

39. Cordes D, Turski PA, Sorenson JA (2000) Compensation of susceptibility-induced signal loss in echo-planar imaging for functional applications. Magn Reson Imaging 18:1055-1068

40. Biswal B, Zerrin Yetkin F, Haughton VM, Hyde JS (1995) Functional connectivity in the motor cortex of resting human brain using echo-planar MRI. Magn Reson Med 34:537-541

41. Griswold MA, Jakob PM, Heidemann RM, Nittka M, Jellus V, Wang J, Kiefer B, Haase A (2002) Generalized autocalibrating partially parallel acquisitions (GRAPPA). Magn Reson Med 47:1202-1210

42. Polimeni JR, Bhat H, Witzel T, Benner T, Feiweier T, Inati SJ, Renvall V, Heberlein K, Wald LL (2016) Reducing sensitivity losses due to respiration and motion in accelerated echo planar imaging by reordering the autocalibration data acquisition. Magn Reson Med 75:665-679

43. Goebel R (2012) BrainVoyager-past, present, future. Neuroimage 62:748-756

44. Witt ST, Laird AR, Meyerand ME (2008) Functional neuroimaging correlates of finger-tapping task variations: an ALE metaanalysis. Neuroimage 42:343-356

45. Li T, Kastrup A, Takahashi AM, Moseley ME (1999) Functional MRI of human brain during breath holding by BOLD and FAIR techniques. Neuroimage 9:243-249

46. Hsu Y, Kuan W, Lim K, Liu H (2010) Breathhold-regulated blood oxygenation level- dependent (BOLD) MRI of human brain at 3 Tesla. J Magn Reson Imaging 31:78-84

47. Ma Y, Berman AJL, Pike GB (2016) The effect of dissolved oxygen on the relaxation rates of blood plasma: implications for hyperoxia calibrated BOLD. Magn Reson Med 76:1905-1911

48. Nakada K, Yoshida D, Fukumoto M, Yoshida S (2001) Chronological analysis of physiological T2* Signal change in the cerebrum during breath holding. J Magn Reson Imaging 13:344-351

49. Thomason ME, Burrows BE, Gabrieli JDE, Glover GH (2005) Breath holding reveals differences in fMRI BOLD signal in children and adults. Neuroimage 25:824-837
50. Birn RM, Diamond JB, Smith MA, Bandettini PA (2006) Separating respiratory-variation-related fluctuations from neuronalactivity-related fluctuations in fMRI. Neuroimage 31:1536-1548

51. Birn RM, Smith MA, Jones TB, Bandettini PA (2008) The respiration response function: the temporal dynamics of fMRI signal fluctuations related to changes in respiration. Neuroimage 40:644-654

52. Talairach J, Tournoux P (1988) Co-planar stereotaxic atlas of the human brain. 3-Dimensional proportional system: an approach to cerebral imaging. Thieme, New York

53. Genovese CR, Lazar NA, Nichols T (2002) Thresholding of statistical maps in functional neuroimaging using the false discovery rate. Neuroimage 15:870-878

54. Lenoski B, Baxter LC, Karam LJ, Maisog J, Debbins J (2008) On the performance of autocorrelation estimation algorithms for fMRI analysis. IEEE J Sel Top Signal Process 2:828-838

55. Jäncke L, Specht K, Mirzazade S, Peters M (1999) The effect of finger-movement speed of the dominant and the subdominant hand on cerebellar activation: a functional magnetic resonance imaging study. Neuroimage 9:497-507

56. Helms G, Dechent P (2009) Increased SNR and reduced distortions by averaging multiple gradient echo signals in 3D FLASH imaging of the human brain at 3T. J Magn Reson Imaging 29:198-204

57. Poser BA, Koopmans PJ, Witzel T, Wald LL, Barth M (2010) Three dimensional echo-planar imaging at 7 Tesla. Neuroimage 51:261-266

58. Evans AC, Collins DL, Mills SR, Brown ED, Kelly RL, Peters TM (1993) 3D statistical neuroanatomical models from 305 MRI volumes. In: Proc. IEEE - Nuclear Science Symposium and Medical Imaging Conference, pp 1813-1817

59. Talagala SL, Sarlls JE, Liu S, Inati SJ (2016) Improvement of temporal signal-to-noise ratio of GRAPPA accelerated echo planar imaging using a FLASH based calibration scan. Magn Reson Med 75:2362-2371

60. Blazejewska AI, Bhat H, Wald LL, Polimeni JR (2017) Reduction of across-run variability of temporal SNR in accelerated EPI time-series data through FLEET-based robust autocalibration. Neuroimage 152:348-359

61. Bodurka J, Ye F, Petridou N, Murphy K, Bandettini PA (2007) Mapping the MRI voxel volume in which thermal noise matches physiological noise-Implications for fMRI. Neuroimage 34:542-549

62. Robinson SD, Pripfl J, Bauer H, Moser E (2008) The impact of EPI voxel size on SNR and BOLD sensitivity in the anterior medio-temporal lobe: a comparative group study of deactivation of the default mode. Magn Reson Mater Phy 21:279-290

63. Brierley B, Shaw P, David AS (2002) The human amygdala: a systematic review and meta-analysis of volumetric magnetic resonance imaging. Brain Res Brain Res Rev 39:84-105

64. Zotev V, Phillips R, Yuan H, Misaki M, Bodurka J (2014) Selfregulation of human brain activity using simultaneous real-time fMRI and EEG neurofeedback. Neuroimage 85:985-995

65. Balteau E, Hutton C, Weiskopf N (2010) Improved shimming for fMRI specifically optimizing the local BOLD sensitivity. Neuroimage 49:327-336

66. Barth M, Breuer F, Koopmans PJ, Norris DG, Poser BA (2016) Simultaneous multislice (SMS) imaging techniques. Magn Reson Med 75:63-81

67. Vu AT, Jamison K, Glasser MF, Smith SM, Coalson T, Moeller S, Auerbach EJ, Ugurbil K, Yacoub E (2017) Tradeoffs in pushing the spatial resolution of fMRI for the 7T human connectome project. Neuroimage 154:23-32

68. Poser BA, Kemper VG, Ivanov D, Uludag K, Kannengiesser SA, Barth M (2013) CAIPIRINHA-accelerated 3D EPI for high 
temporal and/or spatial resolution EPI acquisitions. In: Proc. to ESMRMB Congr. Toulouse, Fr, p 287

69. Narsude M, Gallichan D, van der Zwaag W, Gruetter R, Marques JP (2016) Three-dimensional echo planar imaging with controlled aliasing: a sequence for high temporal resolution functional MRI. Magn Reson Med 75:2350-2361

70. Zahneisen B, Ernst T, Poser BA (2015) SENSE and simultaneous multislice imaging. Magn Reson Med 74:1356-1362

71. Schwarzbauer C, Porter DA (2010) Single shot partial dual echo (SPADE) EPI-an efficient acquisition scheme for reducing susceptibility artefacts in fMRI. Neuroimage 49:2234-2237
72. Tse DHY, Wiggins CJ, Poser BA (2016) High-resolution gradient-recalled echo imaging at 9.4T using 16-channel parallel transmit simultaneous multislice spokes excitations with slice-by-slice flip angle homogenization. Magn Reson Med. doi:10.1002/mrm.26501

73. Pfaffenrot V, Brunheim S, Rietsch SHG, Ernst TM, Orzada S, Quick HH (2017) An 8/15-channel Tx/Rx head neck RF coil combination with semi-dynamic B1 shimming for improved fMRI of the cerebellum at 7 T. In: Proc Intl Soc Mag Reson Med, p 1126 